\title{
Programming effects of peripubertal stress on spatial learning
}

\author{
S. Tzanoulinou ${ }^{\text {a,1 }}$, E. Gantelet ${ }^{\text {a }}$, C. Sandi ${ }^{\text {a }}$, C. Márquez ${ }^{\text {b, }}$ \\ ${ }^{a}$ Laboratory of Behavioral Genetics, Brain Mind Institute, École Polytechnique Fédérale de Lausanne (EPFL), Lausanne, Switzerland \\ ${ }^{\mathrm{b}}$ Laboratory of Neural Circuits of Social Behavior, Instituto de Neurociencias (Universidad Miguel Hernández-Consejo Superior de Investigaciones Científicas), San Juan \\ de Alicante, Spain
}

\section{A R T I C L E I N F O}

\section{Keywords:}

Stress

Peripubertal stress

Water maze

PSA-NCAM

Dentate gyrus

Corticosterone

\begin{abstract}
A B S T R A C T
Exposure to adversity during early life can have profound influences on brain function and behavior later in life. The peripubertal period is emerging as an important time-window of susceptibility to stress, with substantial evidence documenting long-term consequences in the emotional and social domains. However, little is known about how stress during this period impacts subsequent cognitive functioning. Here, we assessed potential longterm effects of peripubertal stress on spatial learning and memory using the water maze task. In addition, we interrogated whether individual differences in stress-induced behavioral and endocrine changes are related to the degree of adaptation of the corticosterone response to repeated stressor exposure during the peripubertal period. We found that, when tested at adulthood, peripubertally stressed animals displayed a slower learning rate. Strikingly, the level of spatial orientation in the water maze completed on the last training day was predicted by the degree of adaptation of the recovery -and not the peak-of the corticosterone response to stressor exposure (i.e., plasma levels at 60 min post-stressor) across the peripubertal stress period. In addition, peripubertal stress led to changes in emotional and glucocorticoid reactivity to novelty exposure, as well as in the expression levels of the plasticity molecule PSA-NCAM in the hippocampus. Importantly, by assessing the same endpoints in another peripubertally stressed cohort tested during adolescence, we show that the observed effects at adulthood are the result of a delayed programming manifested at adulthood and not protracted effects of stress. Altogether, our results support the view that the degree of stress-induced adaptation of the hypothalamuspituitary-adrenal axis responsiveness at the important transitional period of puberty relates to the long-term programming of cognition, behavior and endocrine reactivity.
\end{abstract}

\section{Introduction}

Exposure to adversity during early life can have profound influences on brain function, behavior and cognition at adulthood (Albrecht et al., 2017; Bolton et al., 2017; Sterlemann et al., 2010; Suri et al., 2013), and the precise developmental timing when stress occurs seems to be critical in determining the precise consequences (Gee and Casey, 2015; Lupien et al., 2009). In addition to the recognized impact of neonatal (Bonapersona et al., 2019; Heim and Nemeroff, 2001; Molet et al., 2014; Veenema, 2009) and childhood/juvenile (Albrecht et al., 2017) stress, the peripubertal period is emerging as a time-window of high vulnerability to the programming of emotional (Cordero et al., 2012; Latsko et al., 2016; Márquez et al., 2013; Sheth et al., 2017) and social (Márquez et al., 2013; Poirier et al., 2014; Tzanoulinou et al., 2014a, 2014b) effects of stress (for a review, see (Tzanoulinou and Sandi, 2017).
However, despite the well-known modulatory power of stress on cognition (Lupien et al., 2009; Sandi, 2013), little is known about the impact of peripubertal stress on later life cognitive functioning. A few studies in which stressors were applied during the period expanding from peripuberty till young adulthood have reported enduring learning and memory impairments specifically for the spatial domain (Isgor et al., 2004; Sterlemann et al., 2010). Therefore, whether the peripubertal period per se is susceptible to long-term programming effects of stress on spatial learning, while plausible, remains unclear.

The peripubertal period, involving time-windows right before and after puberty, comprises drastic hormonal, neurobiological and behavioral changes (Andersen and Teicher, 2008; Blakemore, 2008; Casey et al., 2010; Paus et al., 2008; Romeo et al., 2016; Spear, 2000; Tzanoulinou and Sandi, 2016). In particular, this period involves marked changes in the responsivity of the hypothalamic-pituitary-adrenal (HPA)

\footnotetext{
* Corresponding author.

E-mail address: cmarquez@umh.es (C. Márquez).

1 Current address: Department of Biomedical Sciences, University of Lausanne, Lausanne, Switzerland.
} 
axis to stressful experiences (McCormick et al., 2017; Romeo et al., 2016), and this transition can be modified by experiences (Gunnar et al., 2019), particularly stressful ones (Kumsta et al., 2017; Márquez et al., 2013; McCormick et al., 2017; Romeo et al., 2016). Strikingly, individual differences in the adaptation of the glucocorticoid response to repeated stress exposure during the peripubertal period in rats were found to predict subsequent changes in emotional and social phenotypes observed during adolescence (Papilloud et al., 2019) and adulthood (Walker et al, 2017, 2018). In addition, genetic selection in rats for the degree of corticosterone adaptation during peripubertal stress (Walker and Sandi, 2018) underscored genetic line-related differences in spatial learning and memory performance (Huzard et al., 2020). Accordingly, given the strong modulatory capacity of glucocorticoids on brain function and cognition (de Quervain et al., 2017; Sandi, 2011), including spatial learning (Akirav et al., 2004; Conboy et al., 2010; Sandi et al., 1997), we hypothesize that long-term programming of peripubertal stress on spatial learning would depend on the individual degree of glucocorticoid adaptation to repeated stress.

When considering the glucocorticoid adaptation to repeated stress, it is important to distinguish between the peak and the recovery phases, as they serve different adaptive functions (Romeo et al., 2016). While peak glucocorticoid levels facilitate physiological processes to deal with immediate challenges (de Kloet et al., 2008; Myers et al., 2014), the recovery phase (i.e., returning to baseline) is key to protect the organism from maladaptive overactivation and to prepare it for eventual new challenges (Karatsoreos and McEwen, 2011). Importantly, the peripubertal period has been reported to set a change in HPA responsivity in both humans and rats, including changes not only in the peak but also in the recovery phases (McCormick et al., 2017). We have previously reported a strong link between the magnitude of adaptation of the peak corticosterone response to repeated stressors given during the peripubertal period in rats and subsequent changes in emotional and social behaviors (Papilloud et al., 2018; Walker et al, 2017, 2018). However, in the context of the current study on spatial learning, we hypothesize that it will be the adaptation of the recovery phase of corticosterone responsiveness that predicts spatial learning. This hypothesis is based on several premises. First, on the crucial roles of the hippocampus in both, spatial learning (Bird and Burgess, 2008) and in providing negative feedback to the HPA axis (Herman and Mueller, 2006; Jacobson and Sapolsky, 1991; Kovács and Makara, 1988) and, thus, impacting on the corticosterone recovery phase. Second, on the high density of corticosteroid receptors present in the hippocampus (De Kloet, 1991) and their involvement in the HPA axis negative feedback (Reul et al., 1990). Finally, high glucocorticoid levels are known to promote plastic changes in hippocampal structure and function (de Kloet et al., 2018; McEwen et al., 2016), including changes in the expression levels of key plasticity molecules, such as PSA-NCAM (Montaron et al., 2003; Nacher et al., 2004). Importantly, PSA-NCAM -a key post-translational modification of the neural cell adhesion molecule (NCAM)- is critically involved in hippocampal plasticity (Kiss and Muller, 2001) and spatial memory (Bisaz et al., 2009) and modulated by stress (Sandi, 2004).

Therefore, we set this study in rats to assess potential long-term effects of peripubertal stress in spatial learning and memory in the water maze at adulthood, and to investigate whether individual differences in stress-induced changes are related to the adaptation of the corticosterone response (peak vs recovery) to repeated stressor exposure during the peripubertal period. In order to have broader information on the behavioral phenotype for data interpretation, we tested animals in emotional reactivity tasks as well. We also measured plasma corticosterone responses to novelty stress shortly before water maze training to assess both how this response relates to peripubertal corticosterone adaptation and whether it is associated with water maze performance. To understand whether any observed effects at adulthood are the result of a delayed programming or already present shortly after peripubertal stress exposure, we performed a second experiment in which animals were tested during late adolescence. Finally, we assessed levels of the learning and plasticity-related molecule PSA-NCAM in the dentate gyrus (DG) of the hippocampus and, in addition, as a control region, in the medial amygdala (MeA).

\section{Materials and methods}

\subsection{Animals}

Experimental subjects were the male offspring of Wistar Han rats purchased from Charles River Laboratories, France, and bred in our animal facility $(n=70)$. All animals were kept in constant conditions of humidity and temperature $\left(22 \pm 1{ }^{\circ} \mathrm{C}\right)$ with a 12-h light-dark cycle (lights on at 7:00 a.m.). Food and water were available ad libitum. All the procedures described were conducted in conformity with Swiss National Institutional Guidelines on Animal Experimentation, and approved by a license issued from the Swiss Cantonal Veterinary Office Committee for Animal Experimentation.

\subsection{Experimental design}

At weaning (P21), male rats from different litters were distributed into different home cages in groups of two non-siblings, and each cage was randomly assigned to control (CTRL, $n=34$ ) or peripubertal stress (STRESS, $\mathrm{n}=36$ ) conditions. Animals from the STRESS group underwent the peripubertal stress protocol (PPS) starting at P28 (Márquez et al., 2013), and CTRL animals were briefly handled and returned to their home cage. Behavior and hormonal characterizations later in life of the experimental groups were performed at adolescence (P48+) and adulthood (P83+) in independent groups of animals (Fig. 1) (Adolescence CTRL $\mathrm{n}=18$; Adolescence STRESS $\mathrm{n}=18$; Adulthood CTRL $\mathrm{n}=$ 16; Adulthood STRESS $n=18$ ). Before behavioral testing, animals were handled for 3 consecutive days to acclimatize to the experimenter and general conditions. Animals were tested in an Open Field and Novel Object test and two days later, their stress response was assessed after a novelty challenge by measuring corticosterone plasmatic levels (see below). Then, after five days, animals of each experimental group were further divided into two groups, one which would undergo behavioral evaluation of learning and memory in the Morris Water maze (Adolescence CTRL $n=10$; Adolescence STRESS $n=10$; Adulthood CTRL $n=8$; Adulthood STRESS $n=10$ ) and a second one that would be used to study basal levels of polysialylated-neural cell adhesion molecule (PSA-NCAM) in specific brain regions by immunohistochemistry in either adolescence or adulthood (Adolescence CTRL $\mathrm{n}=8$; Adolescence STRESS $\mathrm{n}=8$; Adulthood CTRL $\mathrm{n}=8$; Adulthood STRESS $\mathrm{n}=8$; with one adult animal being excluded from one PSA-NCAM measurement due to poor IHC signal due to quality of the tissue). Animals were sacrificed in basal conditions by transcardial perfusion under anesthesia, brains rapidly removed, post fixed in PFA $4 \%$ for $4 \mathrm{~h}$ and maintained in PBS until further processing for PSA-NCAM immunohistochemistry.

\subsection{Peripubertal stress protocol}

Peripubertal Stress Protocol (PPS) was performed as previously described (Márquez et al., 2013; Veenit et al., 2014). Specifically, the stress protocol consisted of presenting two different fear-inducing stressors (each one lasting $25 \mathrm{~min}$ ): (1) exposure to the synthetic fox odor trimethylthiazoline $(9 \mu \mathrm{l})$ (Phero Tech Inc., Delta, BC, Canada) released through a small cloth, in a plastic box (38 cm length, $27.5 \mathrm{~cm}$ width and $31 \mathrm{~cm}$ height) placed under a bright light (210-250 lx); and (2) exposure to an elevated platform $(12 \times 12 \mathrm{~cm}$, elevated $95 \mathrm{~cm}$ from the ground) under direct bright light (470-500 lx). The stressors were applied subchronically during the peripubertal period (a total of 7 days across postnatal day P28 to P42, i.e., on P28-P30, P34, P36, P40 and P42), during the light phase, and according to a variable schedule, where the order and timing of the stressors were changed on different days (Fig. 2A). On some stress days, only one stressor was presented, 


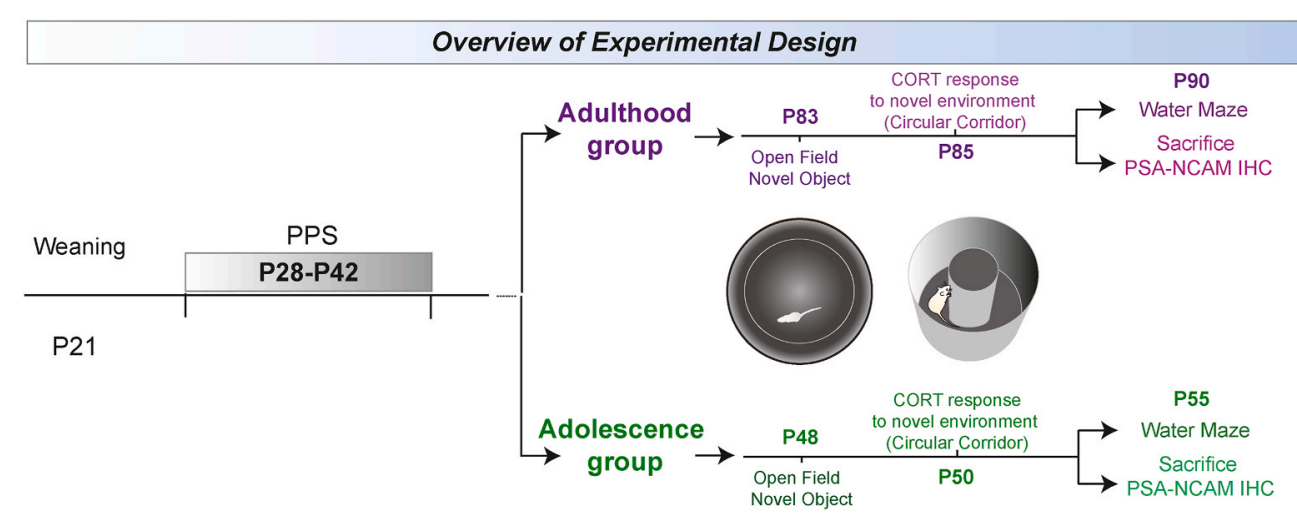

Fig. 1. Overview of experimental design to assess the long-term effects of peripubertal stress (PPS) in different moments of development. Rats were weaned at P21 and were either exposed to the PPS protocol from P28 to P42, or assigned to the Control group. The stressors used were exposure to an elevated platform and to a predator odor (TMT) (for more details, please see materials and methods). Control rats were briefly handled on the days of the PPS and then returned to their home cages. Subsequently, control (CTRL) and peripubertally stressed (STRESS) rats were split in two age groups: the adulthood group, and as a control, the adolescence group, depending on when they underwent further tests. All animals were subjected to an open field and novel object exploration tests. Subsequently, their corticosterone reactivity was evaluated after exposure to a novel environment (i.e. exposure to a circular corridor). They were then further split into a group that performed the water maze and a group that was assessed for PSA-NCAM expression levels in the dentate gyrus and medial amygdala. while on other days, the two stressors were given consecutively. Following each stress session, animals were returned to their home-cages where a transparent Plexiglas wall with holes separated each animal for $15 \mathrm{~min}$ before rejoining their cage mates. On the first and last day of Peripubertal stress, blood samples were collected at different time points only to STRESS animals, in order to study the adaptation dynamics to the stress protocol (see below). The control animals were handled on the days that their experimental counterparts were exposed to stress. Animals in the same cage were always assigned to the same experimental group (either CTRL or STRESS).

\subsection{Open field and novel object reactivity tests}

Rats' exploration levels were assessed in the open field test as previously described (Salehi et al., 2010). They were individually placed in the center of the open field arena (a circular open arena with a diameter of $100 \mathrm{~cm}$ ) and their behavior while freely exploring was monitored for 10 min using a video camera mounted on the ceiling above the center of the arena. For analysis, the floor was divided into three virtual concentric parts, with a center zone in the middle of the arena $(20 \mathrm{~cm}$ diameter), an interior zone (60-cm diameter), and an exterior zone made up of the remaining area along the sidewalls. Different parameters were evaluated with the video tracking system: distance moved (centimeters) and time spent (seconds) in each zone. Immediately after the open field test, rats were submitted to the novel object reactivity (NOR) test. For this purpose, a small, white plastic bottle was placed into the center of the open field while the rat was inside. Rats were then given 5 min to freely explore the novel object. The time spent exploring (touching) the novel object was recorded manually from the video recordings. Moreover, different parameters were evaluated with the video tracking system: time spent (seconds) in the center (where the novel object was placed) and the periphery of the compartment, number and latency of entries to the center, total distance moved (centimeters) in the center and in the whole compartment.

\subsection{Water maze}

In order to test spatial learning and memory, a round black Plexiglas tank with a diameter of $2 \mathrm{~m}$ and a height of $45 \mathrm{~cm}$ was used. The pool was filled with water each day and the temperature was maintained at $25^{\circ} \mathrm{C} \pm 1{ }^{\circ} \mathrm{C}$ during the experiment. A circular platform was submerged
$1.3 \mathrm{~cm}$ below the water surface. The water maze was surrounded by clearly discernible visual cues to facilitate spatial orientation during the training phase. The experiment was divided in two phases: training and probe trial. The training phase lasted from Day 1 to Day 3 and it involved $4 \times 90$-s trials/day/rat with a $30 \mathrm{~s}$ inter-trial interval. The platform remained constantly at the quadrant assigned as the target quadrant. The starting point for each trial was pseudo-randomly chosen. In order to assess the spatial memory of the animals, a probe trial was performed $24 \mathrm{~h}$ after the last training session (Day 4). During this phase, the platform was removed and rats were allowed to swim freely for $90 \mathrm{~s}$. The distance that the animals swam to find the platform was used as an indication of learning. The percentage of the time spent in the quadrant that contained the platform during training (target quadrant) versus the adjacent quadrant was used as an index of spatial memory.

\subsection{Corticosterone responsiveness}

Individual responsiveness to PPS was evaluated in the STRESS group by measurement of plasmatic corticosterone (CORT) levels during the first and last day of PPS exposure. Blood samples were obtained by tailnick protocol (100 $\mu \mathrm{l}$ for peripubertal animals) within 2 min while gently holding the animals with a cloth and, then, animals were returned to their home cage. The tail-nick procedure allows for the collection of blood samples at different time points from the same animal (Márquez et al., 2004), which enables the study of hormonal dynamics. Samples were obtained in basal conditions, immediately following the termination of the elevated platform stress and 30 and $60 \mathrm{~min}$ after the elevated platform stress. Based on these CORT measurements, two adaptation indices were then calculated: time 0 (t0) and recovery 60 (r60). The t0 index reflected the change of the CORT response, immediately after exposure to the stressor, between the last (P42) and the first (P28) day of the protocol (CORT P42 immediately after stress *100/CORT P28 immediately after stress), and thus, expressed a proxy for the adaptation of the initial response to the stressor after subchronic stress. In a similar way, the recovery 60 (r60) index, was calculated to assess recovery adaptation to basal corticosterone levels after exposure to stress (CORT P42 60 min after termination of stress exposure * 100/CORT P28 60 min after termination of stress exposure). Two animals, one from the adolescent group and one from the adulthood group were excluded from all analyses as the values for these variables were exceeding 3 Standard Deviations from the mean. 


\section{A}

\section{Peripubertal Stress Protocol}

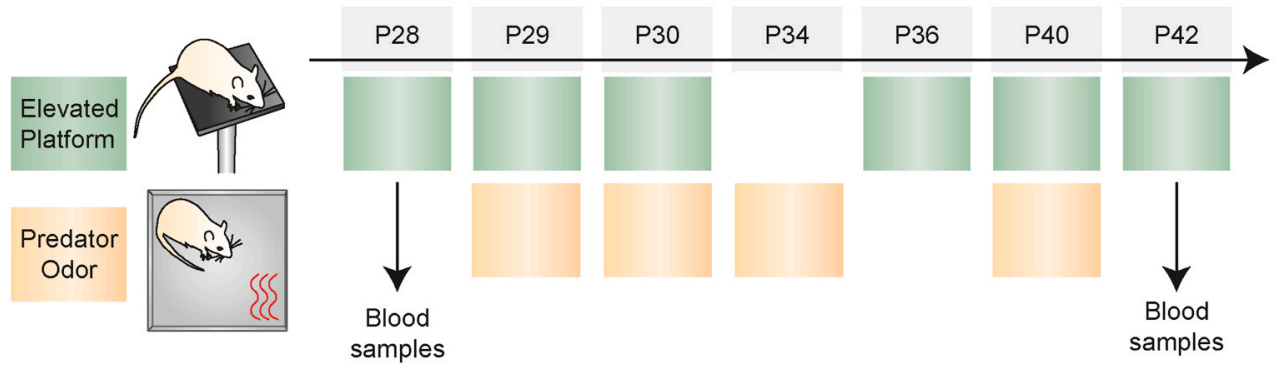

Corticosterone response to peripubertal stressors
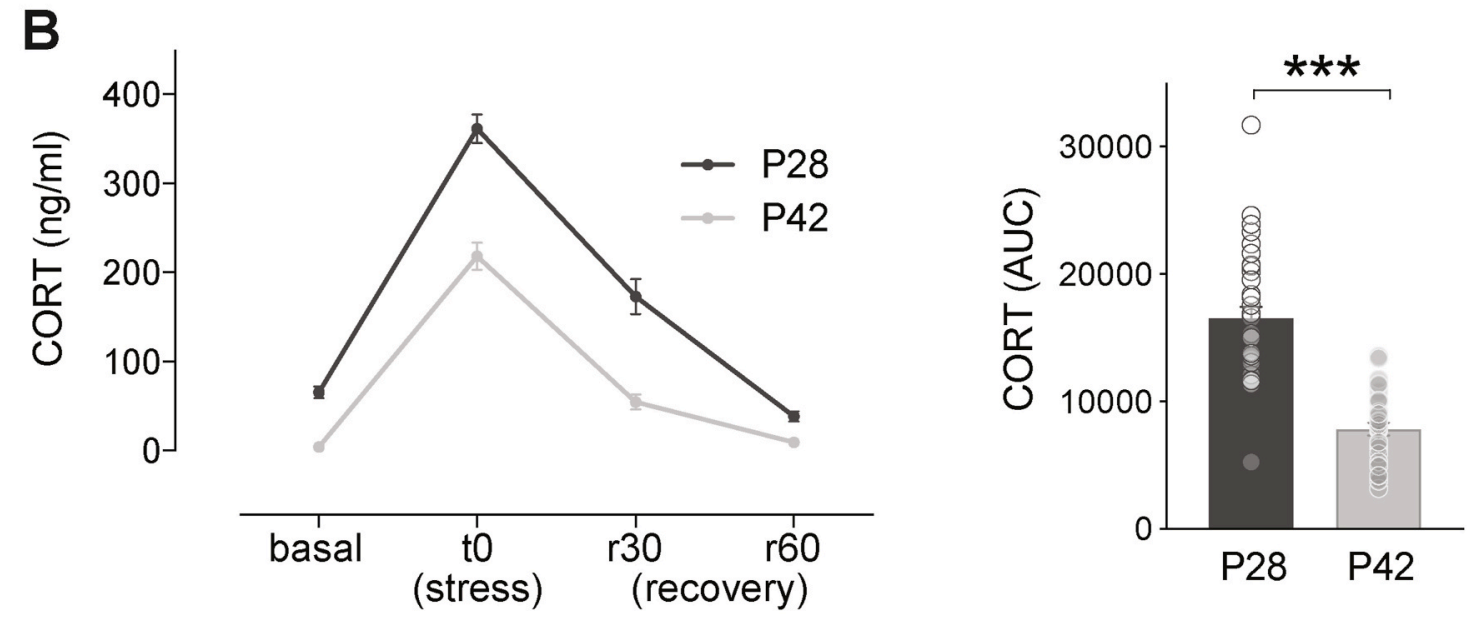

Response immediately after stress - to

Response 60 minutes after stress - $r 60$

C
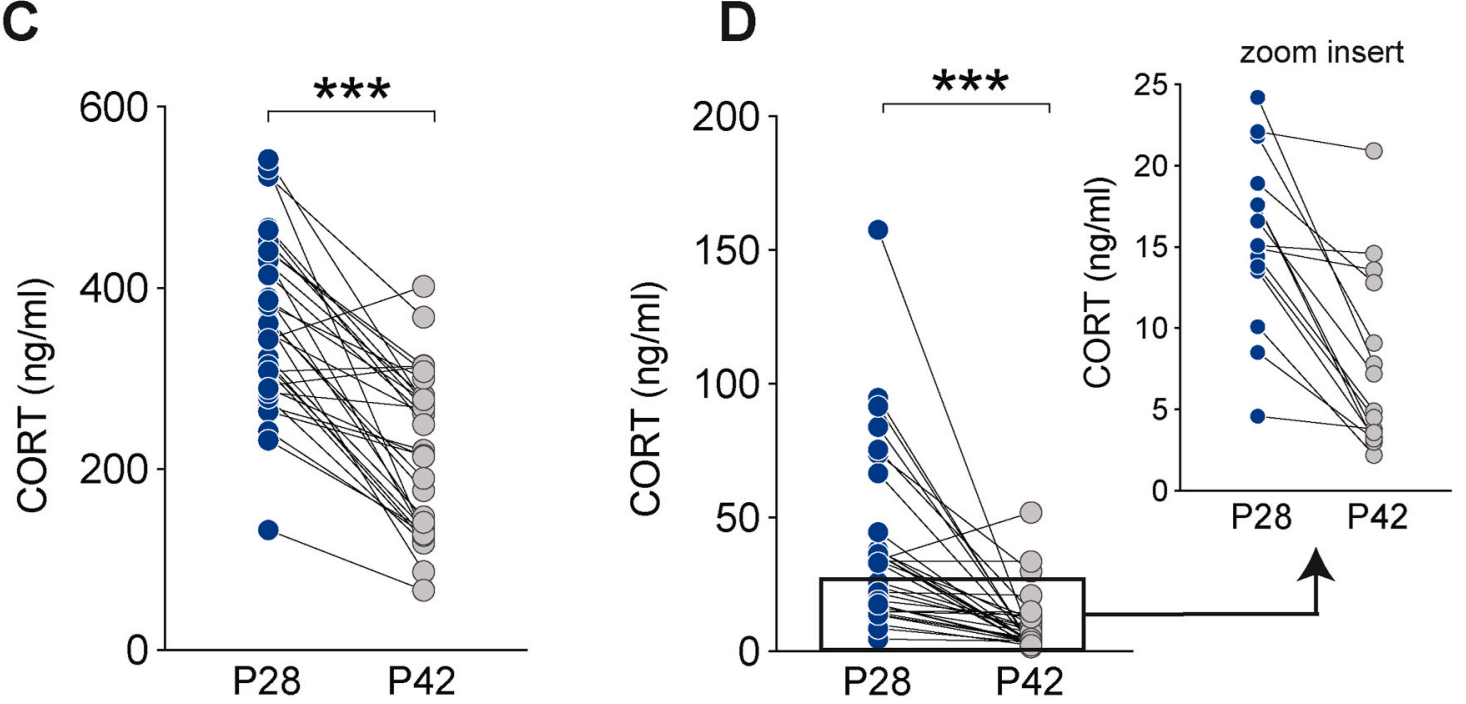

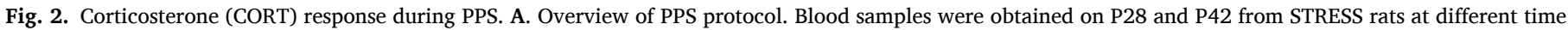

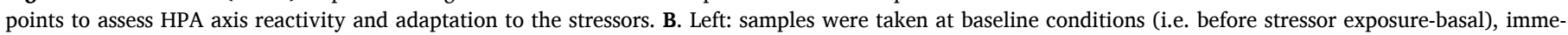

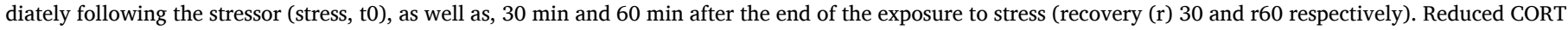

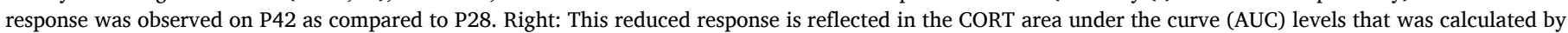

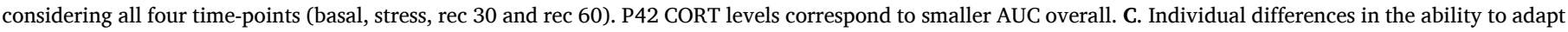

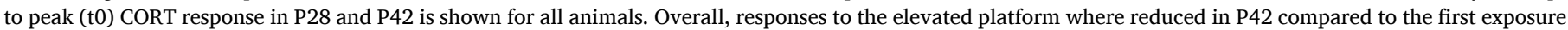

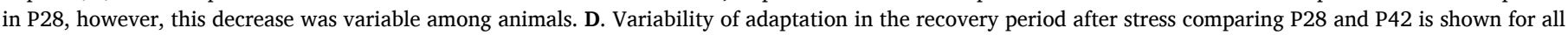

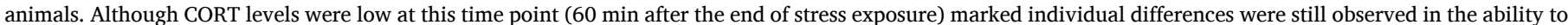
adapt to the subchronic stress. Results are expressed as the mean \pm S.E.M. ${ }^{* * *} \mathrm{p}<0.001$. 
Later in life, the long-term effects of PPS on corticosterone reactivity to a mild stressor were evaluated in independent groups of CTRL and STRESS animals at either adolescence or adulthood period. Immediately after 30 min exposure to a novel environment (a circular corridor made of plastic; $35 \mathrm{~cm}$ high, $25 \mathrm{~cm}$ diameter) blood samples were obtained by tail-nick $(250 \mu \mathrm{l})$. Two additional blood samples were obtained during the recovery period, 30 and $60 \mathrm{~min}$ after the end of circular corridor exposure. Baseline samples were collected in a previous day, in order not to interfere with behavior. Animals from the same home-cage were simultaneously tested in adjacent containers. The containers were cleaned with $1 \%$ acetic acid and dried properly before placing the animals.

Blood samples were collected into ice-cold heparin capillary tubes (Sarsted, Switzerland) and kept at $4^{\circ}$ during the experiment. Plasma was obtained after blood centrifugation at 10,000 rpm for $25 \mathrm{~min}$ and stored at $-20{ }^{\circ} \mathrm{C}$ until analyses. Plasma corticosterone levels were measured by enzymatic immunoassay kit (Correlate-EIA from Assay Designs Inc., USA) according to supplier's recommendations. The area under the curve of the corticosterone levels was calculated using GraphPad Prism (version 7), which computes the area under the curve using the trapezoid rule.

\subsection{PSA-NCAM immunohistochemistry}

For the PSA-NCAM immunohistochemistry experiment rats were anesthetized with a lethal dose of pentobarbital (Esconarkon, Streuli Pharma AG, $150 \mathrm{mg} / \mathrm{kg}$ body weight, solution provided by the EPFL veterinarian) and perfused via the ascending aorta with ice-cold $0.9 \%$ saline, followed by $4 \%$ paraformaldehyde in phosphate-buffered saline ( $\mathrm{pH}=7.5$ ). After perfusion-fixation, the brains were removed from the skull, post-fixed in the same solution for $4 \mathrm{~h}$, and stored in $4{ }^{\circ} \mathrm{C}$ PBS until further processing. Subseries of $50 \mu \mathrm{m}$ thick sections from each group of animals were processed free floating for immunohistochemistry using the avidin-biotin-peroxidase (ABC) method (Hsu et al., 1981). Sections were incubated with $10 \% \mathrm{H}_{2} \mathrm{O}_{2}$ in phosphate buffered saline (PBS) for $10 \mathrm{~min}$ to block endogenous peroxidase activity. They were then treated for $1 \mathrm{~h}$ with $10 \%$ normal donkey serum (NDS) (Jackson ImmunoResearch Laboratories) in PBS with $0.2 \%$ Triton-X100 (Sigma-Aldrich) and incubated for $60 \mathrm{~h}$ at $4{ }^{\circ} \mathrm{C}$ in the primary antibody anti-PSA-NCAM, generated in mouse, (DSHB, 1:1500) with PBS containing 0.2\% Triton$\mathrm{X}-100$. Then, sections were incubated for $2 \mathrm{~h}$ at RT with the biotinylated secondary antibody: donkey anti-mouse IgG (Jackson ImmunoResearch Laboratories, 1:200), followed by an avidin-biotinperoxidase complex (ABC; Vector Laboratories) for $1 \mathrm{~h}$ in PBS. Color development was achieved by incubating with $3,3^{\prime}$-diaminobenzidine tetrahydrochloride (DAB; Sigma-Aldrich) and $0.033 \% \mathrm{H}_{2} \mathrm{O}_{2}$ for $4 \mathrm{~min}$. Finally, sections were mounted on slides, dried for 1 day at room temperature, dehydrated with ascending alcohols and rinsed in xylene. Sections were coverslipped using Eukitt mounting medium (PANREAC). All sections passed through all procedures simultaneously in order to minimize any difference from the immunohistochemical staining itself. To avoid any bias in the analysis, all slides were coded prior to analysis and remained so until the experiment was completed. Sections were examined with an Olympus CX41 microscope under bright-field illumination, homogeneously illuminated and digitalized using a CCD camera. Photographs of the different areas were taken at $20 \AA$ magnification. Grey levels were converted to optical densities (OD) using Image $\mathrm{J}$ software (NIH). Means were determined for each experimental group and data were analyzed with appropriate statistical tests.

\subsection{Statistical analyses}

During behavioral testing animals were tracked automatically with EthoVision 3.0/3.1 (Noldus, Wageningen, the Netherlands). The results were analyzed using the SPSS 17 statistical package and the graphs and correlation matrices were made using GraphPad Prism 7. The data were analyzed with analysis of variance (ANOVA) with repeated measures, Student's $\mathrm{t}$-tests or paired samples $\mathrm{t}$-tests as considered appropriate. The data was checked for distribution with the Shapiro-Wilk test and when the normality was violated, non-parametric tests were applied (i.e. Mann-Whitney and Wilcoxon signed rank tests). All t-tests and paired samples t-tests were performed two-tailed, with the exception of the Mann-Whitney for Fig. 3I and J, where we specifically hypothesized a blunted CORT response (i.e., one-tailed prediction) extrapolating from previous findings (Veenit et al., 2013). Regarding the repeated measures ANOVA, when Mauchly's test of sphericity was significant, thus sphericity could not be assumed, the Greenhouse-Geisser correction was used and reported. All results represent the mean + the standard error of the mean (SEM) and the significance was set at $\mathrm{p}<0.05$.

\section{Results}

\subsection{Marked individual differences in the corticosterone adaptation to repeated stressor exposure during the peripubertal period}

In order to determine whether adaptation to the PPS protocol (peak vs recovery) could predict long term reprogramming effects of stress, we first characterized the corticosterone (CORT) response dynamics during stress exposure. Rats were exposed to threatening challenges (i.e., elevated platform, predator odor) at scattered days (i.e., P28, P29, P30, P34, P36, P40 and P42) within the peripubertal period (Fig. 2A) and blood samples collected following exposure to the same stressor, elevated platform, on the first (P28) and last (P42) days of the protocol, and at four time points: immediately before the stressor (basal), immediately after the elevated platform exposure (stress; t0) and in order to assess the recovery of the response, 30 and $60 \mathrm{~min}$ following the stressor (rec30 and rec60 respectively). In both days, exposure to the elevated platform induced a robust corticosterone release that was recovered to basal levels $1 \mathrm{~h}$ after stress termination (Fig. 2B; left). A general habituation of the CORT response from P28 to P42 was observed, with CORT levels being reduced at $\mathrm{P} 42$, as indicated by a decreased AUC measurement (Fig. 2B; right: $t(32)=9.398, p<0.001$ ). Importantly, inspection of these results indicates that rats displayed marked individual differences in their corticosterone adaptation to peripubertal stress, both as in their peak responses (Fig. 2C) and in the rec 60 time-point (Fig. 2D). Thus, while the majority of animals showed a decreased CORT response at $\mathrm{P} 42$ with varying levels of intensity, suggesting a good degree of adaptation, a subset of rats did not adapt at all (Fig. 2C-D). As expected, the same pattern of CORT response was obtained when animals - ascribed to the two testing conditions later in life - were analyzed separately for validation purposes (Fig. S1A-D; adulthood: A - left; Wilcoxon signed rank paired test - basal: $p<0.001$, stress: $p=0.001$, rec 30: $p<0.001$, rec 60: $\mathrm{p}<0.001$, A - right; $t(16)=7.817, p<0.001$ ), adolescence: $\mathrm{C}$ - left; Wilcoxon signed rank paired test - basal: $p<$ 0.001, stress: $p=0.001$, rec 30: $p=0.004$, rec 60: $p=0.002$, C - right; $t$ $(15)=5.830, p<0.001$, B and D; animals plotted individually for t0 and rec60 time points for adulthood and adolescence respectively. Animals showing low adaptation can be observed in both age groups).

\subsection{Peripubertal stress leads to delayed programming effects on anxiety- like behavior}

Before assessing for potential programming effects of peripubertal stress in spatial learning, we tested animals for their locomotor and exploratory behaviors in the Open Field and Novel Object tests, as behavioral changes in these tests may help interpreting potential differences in the water maze. When tested at adulthood, STRESS rats showed a decrease in the time spent in the center of the Open Field (Fig. 3A; Mann-Whitney $-p=0.014$ ), no differences in total distance moved (Fig. 2B; $t(31)=-0.940, p=0.354$ ), but an increase in selfgrooming behavior (Fig. 3C; Mann-Whitney test, $p=0.007$ ). In addition, STRESS rats showed a trend towards increased time exploring and 

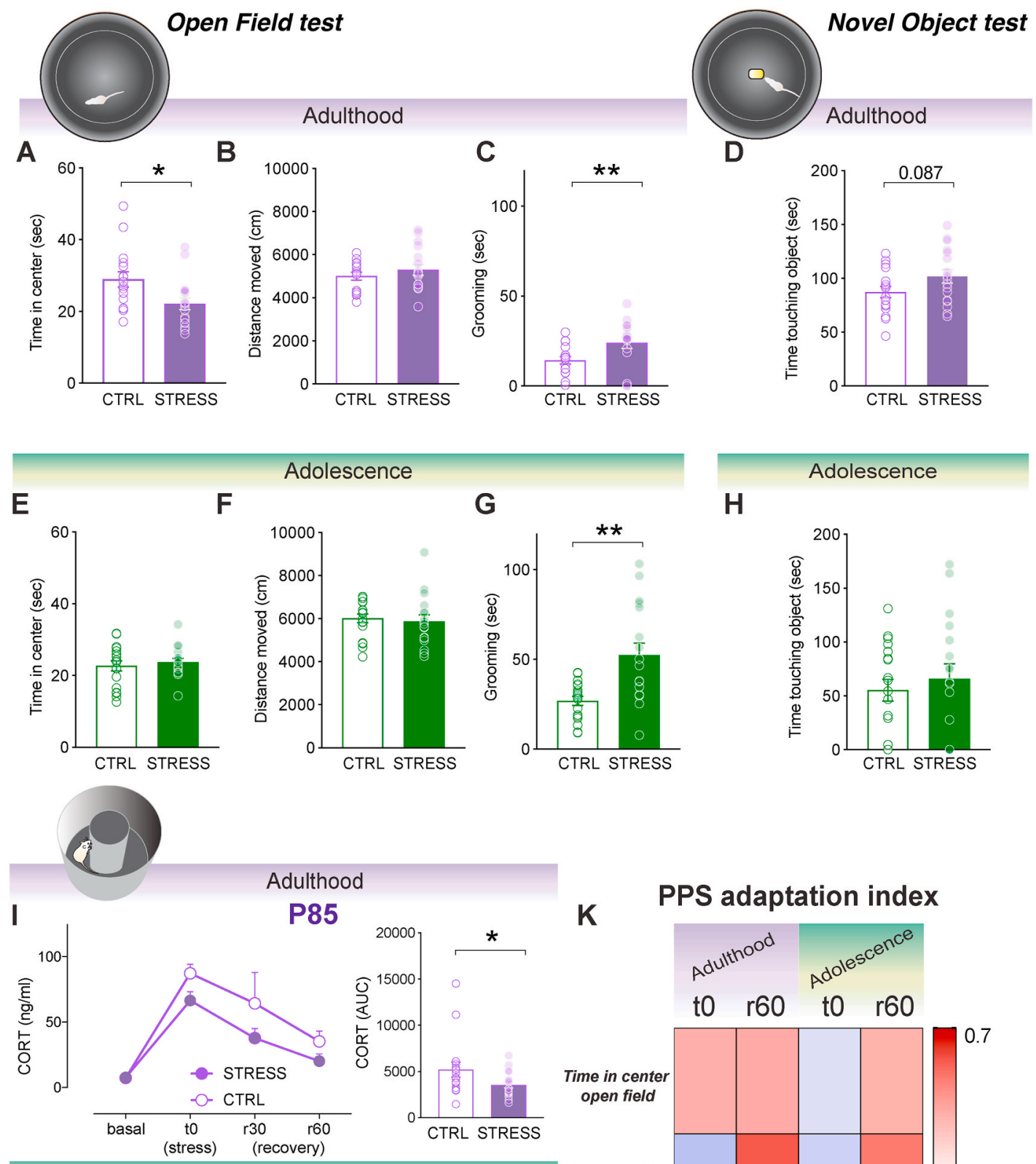

\section{PPS adaptation index}
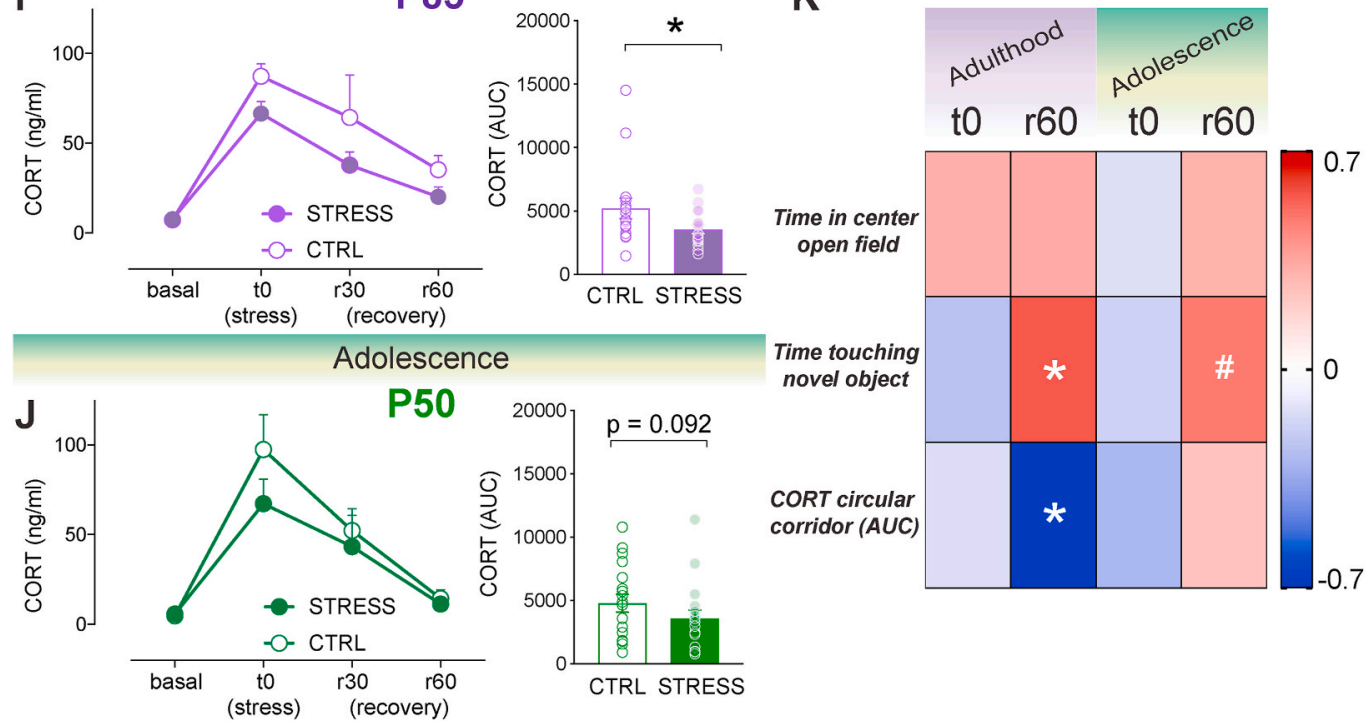

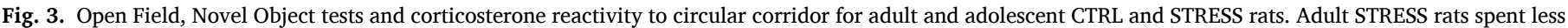

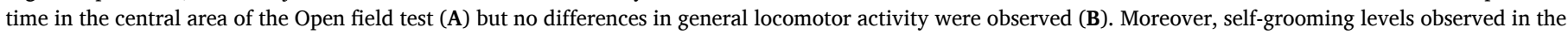

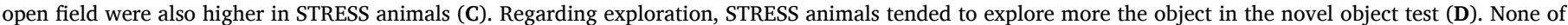

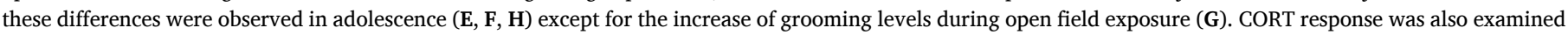

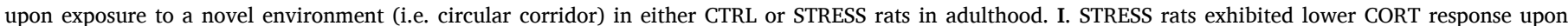

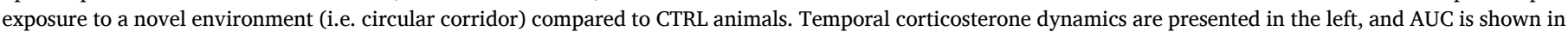

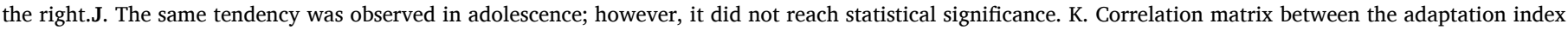

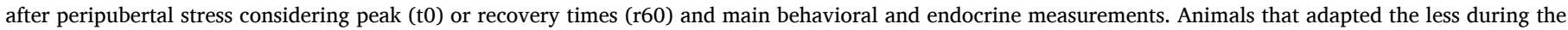

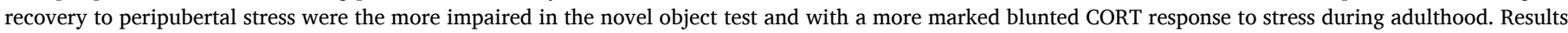
are expressed as the mean \pm S.E.M. ${ }^{*} \mathrm{p}<0.05,{ }^{*} \mathrm{p}<0.01,{ }^{*} \mathrm{p}<0.10$. 
touching the object in the novel object test (Fig. 3D; $\mathrm{t}(31)=-1.769, p$ $=0.087$ ). Altogether, these results indicate a phenotype characterized by increased anxiety-like behaviors with no change in locomotion.

In order to ascertain whether these behavioral changes emerged at adulthood or were already present at earlier time points, we tested a second cohort of animals during adolescence (P48+; Fig. 1). At this time point, STRESS animals did not show changes in the time spent in the center (Fig. 3E; $t(33)=-0.606, p=0.548$ ) or distance moved (Fig. $2 \mathrm{~F} ; t$ $(33)=0.366 . p=0.717)$ in the open field. However, as when tested at adulthood, STRESS animals tested at adolescence showed increased selfgrooming behavior (Fig. 3G; $t(20.434)=-3.584, p=0.002)$. In the novel object test (Fig. 3D; $\mathrm{t}(31)=-1.769, p=0.087$ ), they did not differ from CTRL in time exploring the object (Fig.3H; $t$ (33) $=-0.626, p=$ 0.535).

Therefore, these data indicate an interesting age-dependent effect on exploratory behaviors. Specifically, long-term programming effects of peripubertal stress on anxiety-like behaviors are observed at adulthood, and in a much lesser extent (i.e., self-grooming) at adolescence. Locomotion is not changed at any of the testing times.

\subsection{Peripubertal stress induces CORT hypo-reactivity in adulthood}

We then sought to ascertaining if exposure to PPS would affect corticosterone reactivity to challenges later in life. Indeed, adult STRESS rats showed blunted CORT reactivity (Fig. 3I - left: significant main effect of time: $F(1.4,43.68)=11.434, p<0.001$, trend for a main effect of stress: $F(1,31)=3.861, p=0.058$, nonsignificant stress $\times$ time interaction: $F(1.4,43.68)=0.152, p=0.783,3 \mathrm{I}$ - right: significant MannWhitney one tail test, $p=0.0265$ ) following exposure to a novel environment (i.e., circular corridor, devoid of the anxiogenic center of the arena). This effect was particularly obvious at adulthood, as a mild reduction in CORT activation observed when STRESS animals were tested during adolescence was not significant (Fig. 3J - left; significant main effect of time: $F(1.5,49.05)=14.565, p<0.001$, nonsignificant main effect of stress: $F(1,33)=1.547, p=0.222$, nonsignificant stress $\times$ time interaction: $F(1.5,49.05)=0.615, p=0.498,3 \mathrm{~J}-$ right: nonsignificant Mann-Whitney one tail test, $p=0.092$ ).

Then, we aimed to understand whether the degree to which animals adapt their corticosterone responses to repeated stressors during the peripubertal period (i.e., from P28 to P42) relates to subsequent behavioral and/or hormonal responses. To this end, we first computed two adaptation indices for time 0 (t0) and recovery 60 (r60) (see Methods for details). Then, we calculated correlations between these indices and key behavioral parameters and corticosterone reactivity (AUC) to emotional challenges (i.e., the tests reported above). As shown in Fig. 3K, it was specifically the adaptation of CORT recovery (rec60 index) during peripubertal stress that correlated with both, time touching the object in the Novel object test (Fig. 3K; $\mathrm{r}=0.558, p=$ $0.020)$ and, negatively, with corticosterone reactivity $(r=-0.670, p=$ 0.003 ) in animals tested at adulthood. Thus, the lesser the adaptation of corticosterone recovery during PPS stress exposure, the higher the time exploring the novel object, and the lower the CORT responsiveness to a mild stressor (i.e. novel environment). A similar trend, although not significant, was observed for the correlation between time touching the novel object and rec60 index for the data from adolescence testing (Fig. 3K; $r=0.481, p=0.059$ ). Strikingly, no correlation was observed between the studied parameters and the PPS CORT adaptation index for t0 (i.e, peak CORT stress responses).

\subsection{Peripubertal stress leads to delayed programming effects on spatial learning}

We then addressed our main question; whether peripubertal stress can have delayed effects on spatial learning and memory, and to what extent any observed effect would be related to the degree of CORT adaptation to repeated stressor exposure during peripuberty. To this end, animals were trained and tested to find a hidden platform in the water maze. Adult STRESS rats showed increased total distance swam to find the hidden platform on day 2 (Fig. 4A; left - day 1 - significant main effect of time: $F(3,48)=4.192, p=0.010$, nonsignificant main effect of stress: $F(1,16)=0.295, p=0.595$, nonsignificant time $\times$ stress interaction: $F(3,48)=0.109, p=0.955$, day 2 - significant main effect of time: $F(3,48)=3.231, p=0.030$, significant main effect of stress: $F(1$, 16) $=5.110, p=0.038$, nonsignificant time $\times$ stress interaction: $F(3$, $48)=0.085, p=0.968$, day 3 - nonsignificant main effect of time: $F(3$, $48)=2.182, p=0.102$, nonsignificant main effect of stress: $F(1,16)=$ 2.923, $p=0.107$, nonsignificant time $\times$ stress interaction: $F(3,48)=$ 0.797, $p=0.502$ ) and increased distance moved when the average performance per session was considered (Fig. 4B; middle - significant main effect of time: $F(2,32)=17.083, p<0.001$, significant main effect of stress: $F(1,16)=5.713, p=0.029$, nonsignificant time $\times$ stress interaction: $F(2,32)=1.049, p=0.362)$. No differences between STRESS and control rats were observed during the probe trial (Fig. 4B; right - adjacent: $t(16)=-0.068, p=0.947$, target: $t(16)=0.068, p=$ 0.946).

In order to inquire whether the observed PPS stress effects on spatial learning at adulthood were protracted or delayed, we tested the second cohort of animals in the water maze during adolescence. However, at this time point, no effect of PPS stress was observed (Fig. 4B; left - no effect in distance moved: day 1 - significant main effect of time: $F(3,54)$ $=3.306, p=0.027$, nonsignificant main effect of stress: $F(1,18)=$ $0.832, p=0.374$, nonsignificant time $\times$ stress interaction: $F(3,54)=$ 1.455, $p=0.237$, day 2 - significant main effect of time: $F(3,54)=$ $5.379, p=0.003$, nonsignificant main effect of stress: $F(1,18)=0.032$, $p=0.860$, nonsignificant time $\times$ stress interaction: $F(3,54)=1.476, p$ $=0.231$, day 3 - significant main effect of time: $F(1.76,31.67)=8.329$, $p=0.002$, nonsignificant main effect of stress: $F(1,18)=0.108, p=$ 0.746 , nonsignificant time $\times$ stress interaction: $\mathrm{F}(1.76,31.67)=0.217$, $\mathrm{p}=0.778$ ) nor regarding the average performance per session (Fig. 4B; middle - significant main effect of time: $\mathrm{F}(2,36)=44.392$, $\mathrm{p}<0.001$, nonsignificant main effect of stress: $F(1,18)=0.058, p=0.812$, nonsignificant time $\times$ stress interaction: $F(2,36)=0.871, p=0.427$, nor in the probe test (Fig. 4B; right - adjacent: $t(18)=1.360, p=0.191$, target: $t(18)=-1.361, p=0.190)$.

We then inquired whether individual differences in CORT adaptation during PPS exposure (i.e., t0 and rec60 indices) related to differences in key parameters of water maze performance. To this end, we selected average performance (i.e., distance to the platform) on the last training day, as an index for the maximal acquisition level obtained and distance to find the platform on the first trial of day 2, as a first long-term memory index. As shown in Fig. 4C, rec60 was again the parameter that showed a positive correlation with day 3 performance (Fig. $4 \mathrm{C} ; \mathrm{r}=0.648, p=$ 0.043); i.e., the lesser the adaptation of CORT recovery following peripubertal stressors, the worse the maximal training performance in the water maze. This was not observed when the animals were tested in adolescence (Fig. 4C). Moreover, no correlations were found between water maze performance and PPS peak adaptation index (t0 index) at any of the age groups (Fig. 4C).

Furthermore, in order to better understand possible links between CORT responsiveness during the testing period and variation in spatial learning performance, we examined the relationship between CORT response upon exposure to the circular corridor (see Fig. 3I and J) and water maze parameters. Interestingly, CORT reactivity to the circular corridor correlated with the first long-term memory test (i.e., distance to find the platform on the first trial of day 2) in STRESS animals tested at adulthood; i.e., the lower the CORT the poorer their performance in the water maze (Fig. 4D; $r=-0.641, p=0.046$ ). In other words, those animals that displayed more blunted corticosterone response during adulthood after a novelty challenge as a consequence of peripubertal stress exposure were the ones showing worst long-term memory in the water maze. A similar trend was observed for STRESS animals' CORT responsiveness at adulthood and average performance on the last 


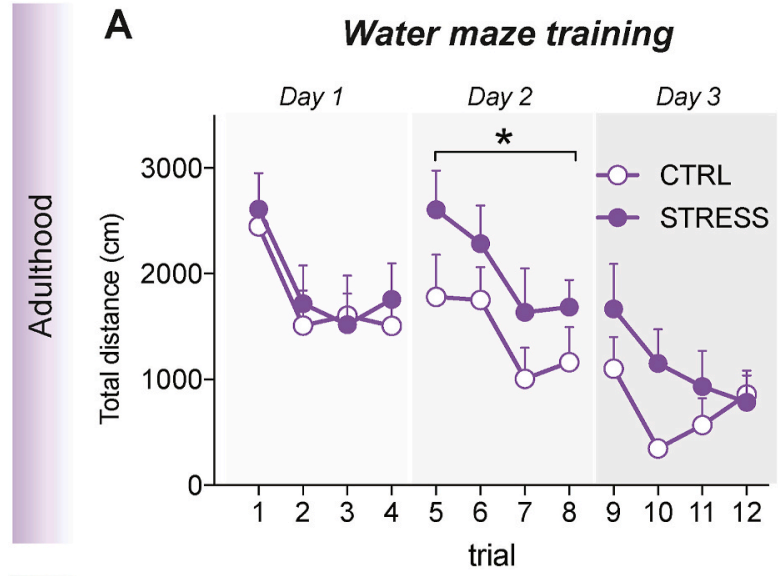

B
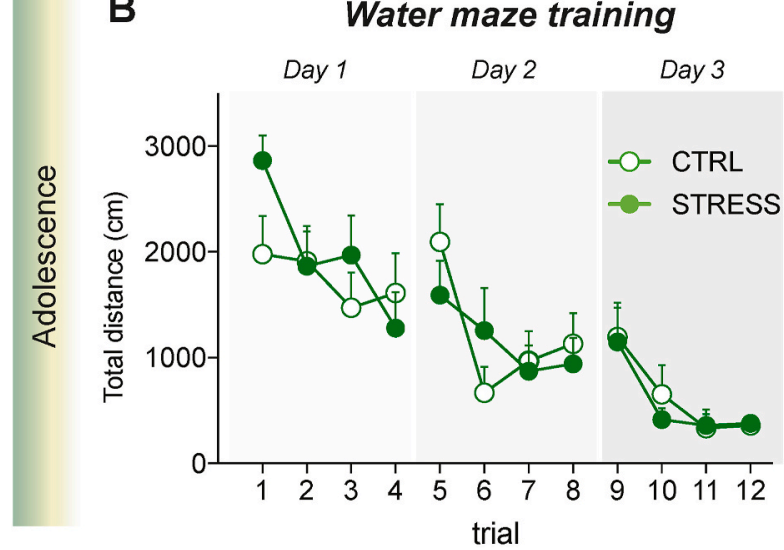

C

PPS adaptation index

\begin{tabular}{|c|r|r|r|}
\hline \multicolumn{2}{|c|}{ Adulthood } & \multicolumn{3}{c|}{ Adolescence } \\
\hline to & r60 & to & r60 \\
\hline & & & \\
\hline & $*$ & & \\
\hline
\end{tabular}

Average performance per session

\section{Probe test}

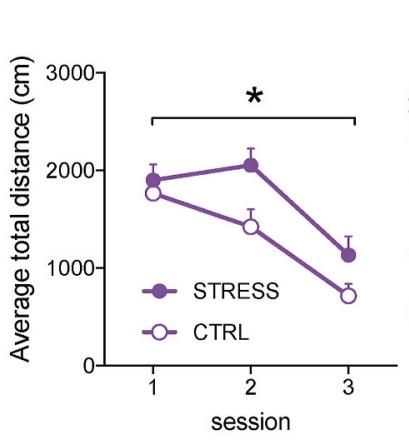

Average performance per session

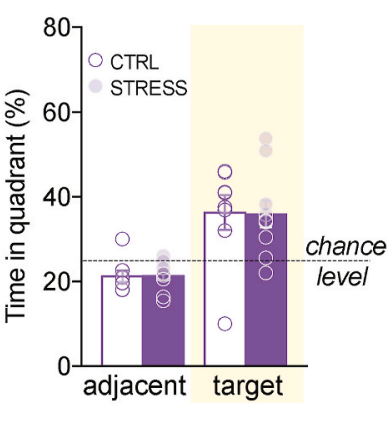

\section{Probe test}
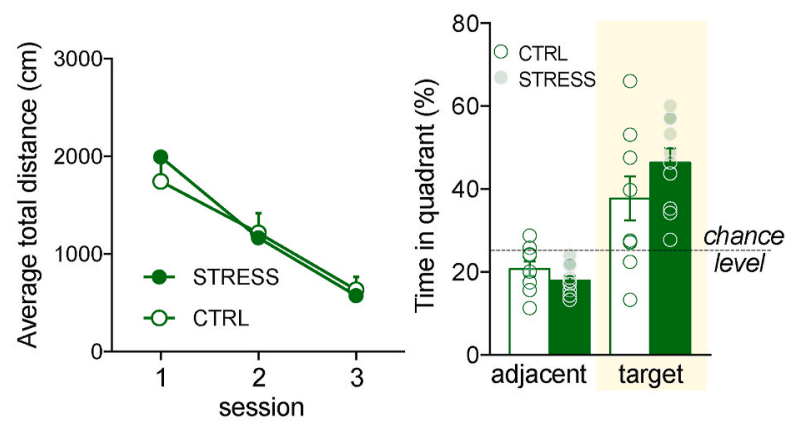

D

\section{Circular Corridor CORT}

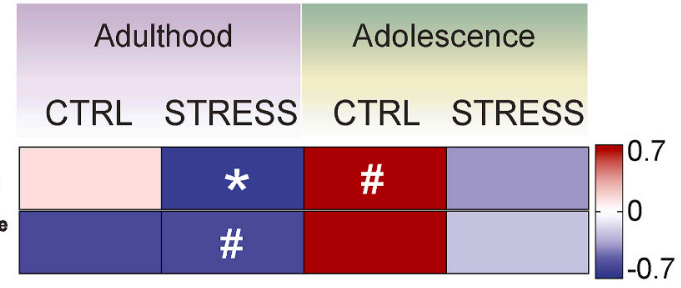

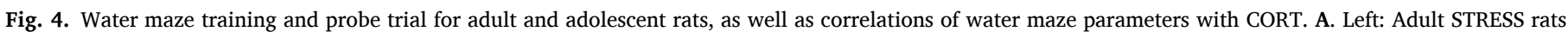

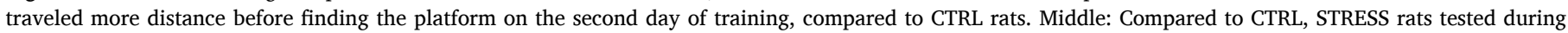

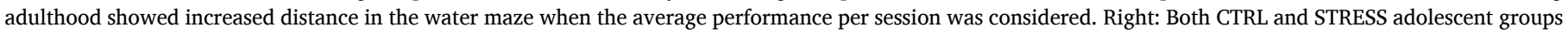

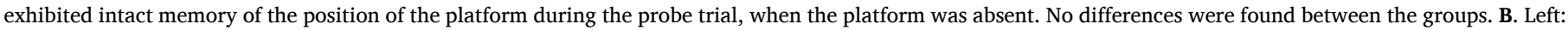

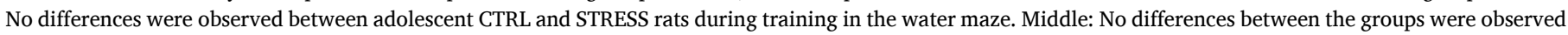

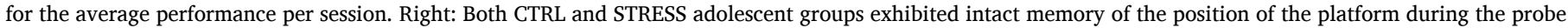

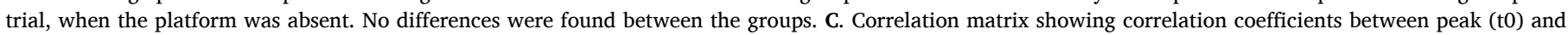

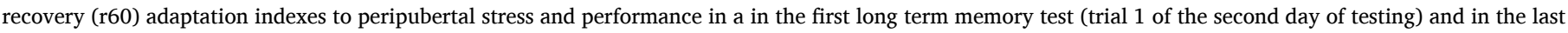

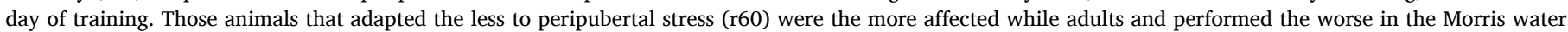

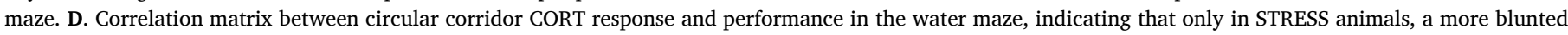

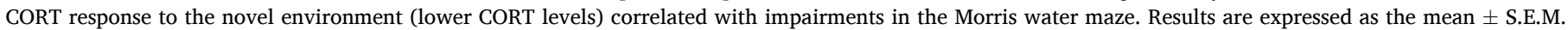
Correlation coefficients ( $\mathrm{r}$ values) in correlation matrix are color-coded. ${ }^{*} \mathrm{p}<0.05, \# \mathrm{p}<0.10$

training day (Fig. 4D; $\mathrm{r}=-0.581, p=0.078$ ).

Altogether, these results suggest that peripubertal stress has delayed detrimental effects on spatial learning that become evident when the assessment happens during adulthood, and that those animals that show impaired adaptation in CORT recovery to repeated stressors exposure perform poorer in a spatial learning task.

\subsection{Peripubertal stress leads to changes in PSA-NCAM in the dentate gyrus}

In order to gain insight into key plasticity molecules, related to learning and memory, that could be affected by peripubertal stress, two further cohorts of rats were exposed to peripubertal stress and studied at each age group (i.e., CTRL and STRESS; Adulthood and Adolescence) and assessed for the expression of PSA-NCAM in the DG of the hippocampus (Fig. 5). As shown in Fig. 5A, there was an increase of PSANCAM in adult rats stressed during peripuberty (Fig. 5A - left; $t$ (12) 


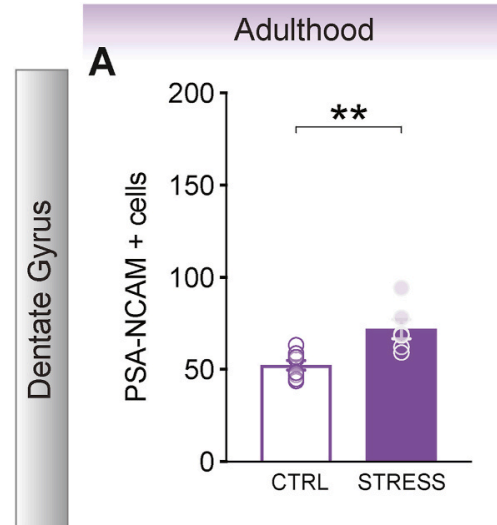

C

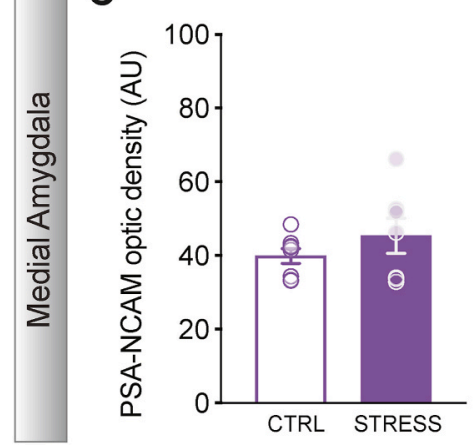

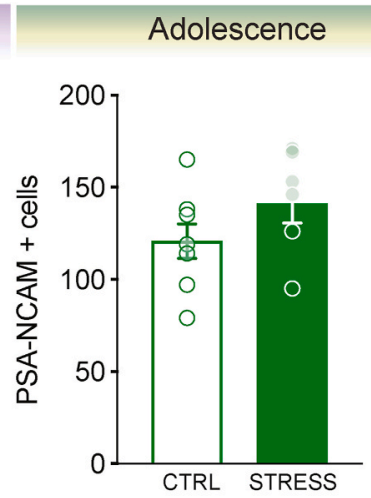

D
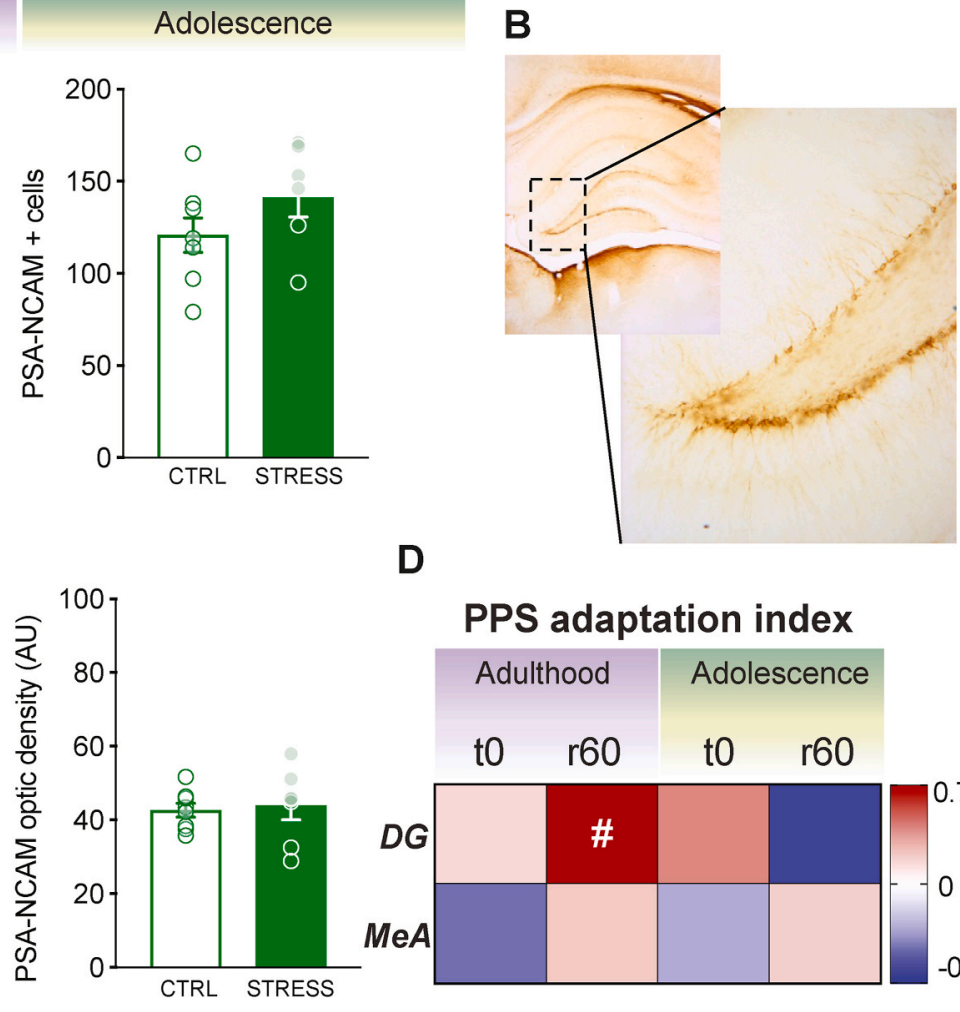

\section{PPS adaptation index}

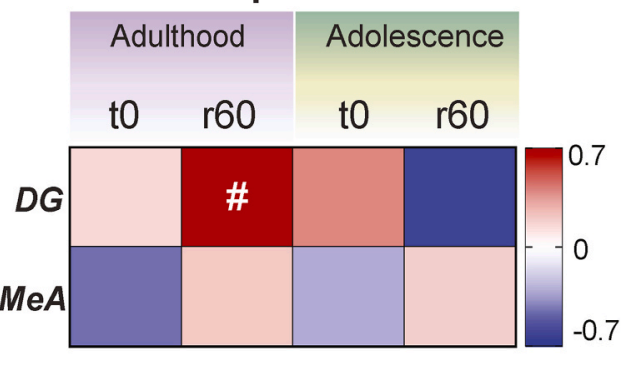

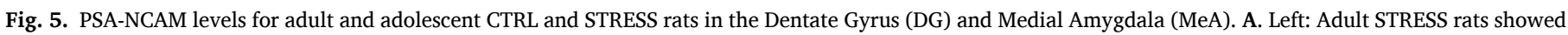

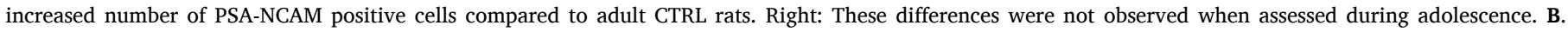

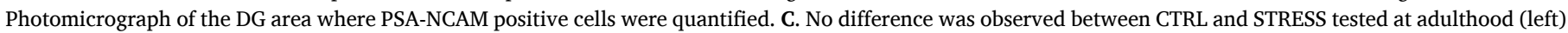

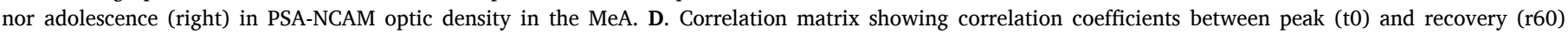

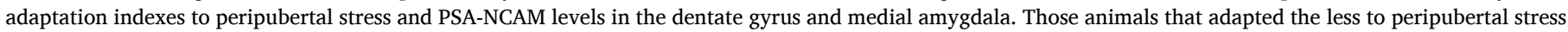

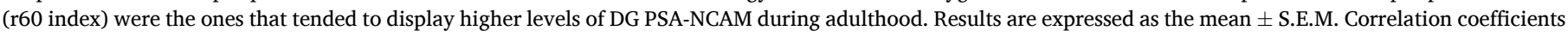
( $\mathrm{r}$ values) in correlation matrix are color-coded. ${ }^{* *} \mathrm{p}<0.01, \# \mathrm{p}<0.10$

$=-3.675, p=0.003)$. However, no significant differences were apparent in PSA-NCAM expression when the rats were assessed during adolescence (Fig. 5A - right; $t(13)=-1.458, p=0.169$ ). In order to study specificity of our findings, we quantified PSA-NCAM expression in the medial amygdala. However, no differences were found between STRESS and CTRL rats regardless of the developmental age, suggesting that the PSA-NCAM alterations observed in the dentate gyrus at adulthood were not only age-dependent, but also brain region-specific (Fig. 5C - left - adulthood; $t(8.04)=-1.059, p=0.321$, right adolescence; $t(13)=-0.291, p=0.776)$.

Interestingly, there was a trend for those animals whose CORT responsiveness would adapt suboptimally at r60 during peripubertal stress to display higher DG PSA levels in adulthood $(\mathrm{r}=0.735 p=0.096)$ (Fig. 5D). No correlations of CORT responsiveness were observed with the MeA for any of the age groups or time points.

\section{Discussion}

Here, we show that exposure to stressors across the peripubertal period in rats leads to cognitive, behavioral and endocrine changes at adulthood. Specifically, peripubertal stress led to impaired spatial learning, increased anxiety-like behavior, and blunted corticosterone responsiveness to novelty challenges. These effects are delayed in nature, as they were not displayed by animals tested during adolescence ( $i$. e., shortly after peripubertal stress exposure). Strikingly, individual differences in the degree of adaptation of the recovery -and not the peakof the corticosterone response to stressor exposure (i.e., plasma levels at 60 min post-stressor) across the peripubertal stress period (i.e., from P28 to $\mathrm{P} 42$ ) predicted the level of spatial orientation in the water maze completed on the last training day, as well as the exploratory behavior shown by animals at adulthood. In addition, this corticosterone stress adaption recovery index (rec60) was inversely related to the corticosterone responsiveness to novelty at adulthood. These findings contribute to further our understanding on the link between HPA axis adaptation to early life stressors at the important transitional period of puberty and the long-term programming of behavior and cognition.

Thus, a main finding of our study is the identification of peripuberty as a stress-sensitivity period for the modulation of adult spatial learning abilities. Previous studies had underscored the early postnatal period as a time-window in which stress exposure makes individuals particularly prone to show spatial learning impairments at long-term life stages (Brunson et al., 2005; Oomen et al., 2010). However, previous studies comprising stressor exposure across several weeks from juvenility to adulthood in which spatial learning and memory impairments were reported (Isgor et al., 2004; Sterlemann et al., 2010) did not allow disentangling the putative impact of peripubertal stress per se. In addition, we show here that the impact is not immediate (i.e., not shown during adolescence) but, similarly to the report by Isgor et al. (2004), it only emerges when testing takes place several weeks after the end of the stress protocol, at adulthood. Further evidence for this delayed phenomenon stems from studies in rats involving prepubertal stress (i.e., from P28 to P30) and showing impaired water maze at adulthood only following a second stressful challenge at adulthood that, on its own, does not affect spatial learning performance (Avital and Richter-Levin, 2005). In this connection, we previously reported that the same peripubertal stress protocol as the one applied here leads as well to attention deficits in adulthood (Tzanoulinou et al., 2016), but whether these deficits are observed already during adolescence remains to be tested. Altogether, 
these findings support the view that the long-term cognitive impact of peripubertal stress requires an incubation period during which stress-targeted mechanisms interact with ongoing maturational and neurodevelopmental trajectories to produce phenotypic changes at later life stages.

Spatial learning highly depends on the functioning of the hippocampus (Moser et al., 1995), a brain region that undergoes profound structural and functional changes in adolescence (McCormick and Mathews, 2010). Interestingly, efficient spatial orientation strategies in the water maze task appear around P42 (Schenk, 1985), coinciding with the last day of our peripubertal stress protocol. Our own data on the expression levels of the plasticity molecule PSA-NCAM in the dentate gyrus show a down-regulation from adolescence to adulthood; this age-dependent regulation was not observed in the medial amygdala, a brain region not involved in spatial learning. Thus, our data agrees with an age-dependent pattern of PSA-NCAM down-regulation taking place in the brain during the postnatal period (Angata and Fukuda, 2003; Rutishauser, 2008) and remaining present later in life in brain areas that maintain neurogenic potential or heightened plasticity, such as the hippocampus (Angata and Fukuda, 2003), where it has been causally involved in memory consolidation (Doyle et al., 1992; López-Fernández et al., 2007; Sandi et al., 2003; Venero et al., 2006). Importantly, we found that peripubertal stress leads to increased PSA-NCAM levels specifically in the dentate gyrus, that was particularly evident in the group of animals examined at adulthood, in agreement with similar findings following exposure to pre-pubertal/juvenile stress (Tsoory et al., 2008). In addition, Tsoory et al. (2008) reported that animals stressed during pre-puberty (P28 to P30) displayed impairments in emotional behavior when exposed to stressful situations later in adulthood, such as the two-way shuttle avoidance. Our results expand this previous observation, and link the ability to adapt to peripubertal stress with corticosterone reactivity to stressors later in life, to cognitive performance and DG PSA-NCAM levels. In the future, it would be interesting to evaluate whether these differences in behavior also translate to changes in corticosterone dynamics after exposure to more severe procedures than the one we used in our study, such as chronic stress or severe acute stress (as the shuttle avoidance used in Tsoory et al., 2008). It might well be that effects of early life stress on HPA axis responsivity might be dependent on the stress intensity, and should be addressed in future studies. Our results reflect the effectiveness of peripubertal stress to disrupt the maturation of the hippocampal learning system. PSA can promote neuronal and synaptic plasticity through mechanisms involving its de-adhesive properties, as well as by interacting with extracellular matrix molecules and glutamate receptors (Varbanov and Dityatev, 2017). However, while PSA-NCAM expression in the dentate gyrus transiently increases around $12 \mathrm{~h}$ following training in the water maze (Murphy et al., 1996; Sandi et al., 2003), this increase is only observed in bad -but not good-learners, that require increased effort to complete the task (Sandi et al., 2004), and it decays as animals progressively master the task (Murphy et al., 1996). Moreover, chronic stress at adulthood leads to increased hippocampal PSA-NCAM expression (Pham et al., 2003; Sandi et al., 2001) and impairs spatial learning in the water maze (Sandi, 2004; Venero et al., 2002). Therefore, the facilitation of learning and plasticity processes by PSA-NCAM seems to require an activity-dependent process triggering a transient increase in its expression. Heightened basal elevation of PSA-NCAM appears to be deleterious to information processing, which aligns with our findings in the current study. Furthermore, we should also note that we found a trend for dentate gyrus PSA-NCAM levels to correlate with the adaptation of the corticosterone recovery levels across the peripubertal stress protocol (rec60 index; i.e., the lower the adaptation, the higher PSA-NCAM levels). Although until further replication these findings should be taken with caution given the reduced sample size, they point towards a potential role of glucocorticoids on the regulation of hippocampal PSA-NCAM expression by peripubertal stress. Indeed, a complex regulation of hippocampal PSA-NCAM by glucocorticoids has been revealed
(Nacher et al., 2004; Rodríguez et al., 1998), involving, in particular, glucocorticoid receptor actions (Montaron et al., 2003).

Importantly, the peripubertal period entails a transition in HPA responsivity to stressors at both, peak and recovery phases (McCormick et al., 2017). Strikingly, we found that individual differences in the spatial orientation levels achieved in the last training day were also related to the peripubertal rec60 corticosterone adaptation index. Thus, those animals that showed a poorer adaptation of the corticosterone stress recovery at puberty were the ones that attained poorer performance levels. Importantly, as hypothesized, it was the corticosterone recovery, and not the peak, index that related to water maze performance. The ability to down-regulate the HPA axis response to stress (and thus, corticosterone levels) following stress exposure through negative feedback is essential to protect the organism from maladaptive overactivation (Karatsoreos and McEwen, 2011) and also important for optimal secretion of corticosterone in basal (unstressed) conditions (Gjerstad et al., 2018). Therefore, our rec60 index seems to have captured individuals' ability to adapt to repeated life stressors and serves as a predictive index of adult life cognitive, behavioral, and endocrine disturbances. These findings align well with the important role of the hippocampus in providing negative feedback to the HPA axis (Herman and Mueller, 2006; Jacobson and Sapolsky, 1991; Kovács and Makara, 1988) and the involvement of hippocampal glucocorticoid receptors in HPA axis negative feedback (De Kloet, 1991; Reul et al., 1990).

In addition, the first index of long-term memory performance (i.e., distance moved to find the platform on the first trial of training day 2) was related to corticosterone reactivity at adulthood. Specifically, animals that showed poorer retention levels on the first trial following training day 1 were the ones that showed blunted corticosterone reactivity when exposed as adults to a novelty challenge. These observations align well with the well-known contribution of training-triggered corticosterone levels for memory function in general (de Kloet et al., 2018; de Quervain et al., 2017; Sandi, 2011) and, specifically, for the consolidation of spatial information (Akirav et al., 2004; Conboy et al., 2010; Huzard et al., 2020; Quirarte et al., 1997; Sandi et al., 1997).

The incubation period reported here for spatial learning effects of peripubertal stress to emerge at adulthood appears to be specific for the cognitive domain. Indeed, a different process seems to be engaged in the development of anxiety-like behaviors. While, as in previous studies (Cordero et al., 2016; Tzanoulinou et al., 2014a), we observe here increased anxiety-like behavior when peripubertally stressed rats were tested at adulthood, decreased anxiety-like behaviors were reported when tested during late adolescence (Toledo-Rodriguez and Sandi, 2011). Furthermore, in the social domain, peripubertal stress leads to increased adult aggression (Márquez et al., 2013) in a protracted manner, as rats that showed aberrant play fighting during adolescence were those that developed a more aggressive phenotype at adulthood (Papilloud et al., 2018). In addition, and in line with its physiological contribution to deal with immediate challenges (de Kloet et al., 2008; Myers et al., 2014), it is the magnitude of adaptation of the peak corticosterone response to peripubertal stress that predicts alterations in emotional and social behaviors (Papilloud et al., 2018; Walker et al, 2017, 2018). Along the same lines, we demonstrated here, that the ability to adapt during recovery periods, i.e. once stress exposure has ended, is a key predicting factor for special learning impairments.

In summary, our study identifies the peripubertal period as a timewindow at which stress can lead to long-term changes in HPA axis reactivity that are related to difficulties in spatial learning abilities later in life. These findings pave the way for further studies to identify mechanisms of both vulnerability and resilience to early trauma. Furthermore, our data suggest that the reprograming effects of early stress might need a period of incubation which could be compensated in young and more plastic brains, but would fail to adapt during adulthood. Accordingly, following early detection of stress-vulnerable individuals, there may be a window of opportunity for therapeutic approaches to act 
during adolescence deflecting the course trajectory towards psychopathology and cognitive impairments.

\section{CRediT authorship contribution statement}

S. Tzanoulinou: Formal analysis, Visualization, Writing - original draft, Writing - review \& editing. E. Gantelet: Investigation, Methodology. C. Sandi: Conceptualization, Writing - review \& editing, Funding acquisition. C. Márquez: Conceptualization, Supervision, Investigation, Methodology, Formal analysis, Visualization, Writing - original draft, Writing - review \& editing, Funding acquisition.

\section{Declaration of competing interest}

The authors declare no competing interests.

\section{Acknowledgements}

We thank Angélique Voucher, Coralie Siegmund, Marjorie Clerc and Aliénor Sonnay for excellent technical assistance.

\section{Appendix A. Supplementary data}

Supplementary data related to this article can be found at https:// doi.org/10.1016/j.ynstr.2020.100282.

\section{Funding}

This work was supported by the NARSAD Young Investigator Grant from the Brain \& Behavior Research Foundation, USA, under the grant number 26478 to C.M., the Ministerio de Ciencia e Innovación, Spain, under the grants RTI2018-097843-B-100 to C.M. and the "Severo Ochoa" Program for Centers of Excellence in R\&D (SEV-2013-0317 and SEV-2017-0723) and by the Swiss National Science Foundation, Switzerland (NCCR Synapsy No. 158776 and 185897) to C.S.; C.M. was further supported by the Ministerio de Ciencia e Innovación with a Ramon y Cajal contract (RYC-2014-16450).

\section{References}

Akirav, I., Kozenicky, M., Tal, D., Sandi, C., Venero, C., Richter-Levin, G., 2004. A facilitative role for corticosterone in the acquisition of a spatial task under moderate stress. Learn. Mem. 11, 188-195. https://doi.org/10.1101/lm.61704.

Albrecht, A., Müller, I., Ardi, Z., Çalışkan, G., Gruber, D., Ivens, S., et al., 2017. Neurobiological consequences of juvenile stress: a GABAergic perspective on risk and resilience. Neurosci. Biobehav. Rev. 74, 21-43. https://doi.org/10.1016/j. neubiorev.2017.01.005.

Andersen, S.L., Teicher, M.H., 2008. Stress, sensitive periods and maturational events in adolescent depression. Trends Neurosci. 31, 183-191. https://doi.org/10.1016/j. tins.2008.01.004.

Angata, K., Fukuda, M., 2003. Polysialyltransferases: major players in polysialic acid synthesis on the neural cell adhesion molecule. Biochimie 85, 195-206. https://doi. org/10.1016/s0300-9084(03)00051-8.

Avital, A., Richter-Levin, G., 2005. Exposure to juvenile stress exacerbates the behavioural consequences of exposure to stress in the adult rat. Int. $\mathrm{J}$. Neuropsychopharmacol. 8, 163-173. https://doi.org/10.1017/ S1461145704004808.

Bird, C.M., Burgess, N., 2008. The hippocampus and memory: insights from spatial processing. Nat. Rev. Neurosci. 9, 182-194. https://doi.org/10.1038/nrn2335.

Bisaz, R., Conboy, L., Sandi, C., 2009. Learning under stress: a role for the neural cell adhesion molecule NCAM. Neurobiol. Learn. Mem. 91, 333-342. https://doi.org/ 10.1016/j.nlm.2008.11.003.

Blakemore, S.-J., 2008. The social brain in adolescence. Nat. Rev. Neurosci. 9, 267-277. https://doi.org/10.1038/nrn2353.

Bolton, J.L., Molet, J., Ivy, A., Baram, T.Z., 2017. New insights into early-life stress and behavioral outcomes. Curr. Opin. Behav. Sci. 14, 133-139. https://doi.org/10.1016/ j.cobeha.2016.12.012.

Bonapersona, V., Kentrop, J., Van Lissa, C.J., van der Veen, R., Joëls, M., Sarabdjitsingh, R.A., 2019. The behavioral phenotype of early life adversity: a 3 level meta-analysis of rodent studies. Neurosci. Biobehav. Rev. 102, 299-307. https://doi.org/10.1016/j.neubiorev.2019.04.021.

Brunson, K.L., Kramár, E., Lin, B., Chen, Y., Colgin, L.L., Yanagihara, T.K., et al., 2005. Mechanisms of late-onset cognitive decline after early-life stress. J. Neurosci. 25, 9328-9338. https://doi.org/10.1523/JNEUROSCI.2281-05.2005.
Casey, B.J., Jones, R.M., Levita, L., Libby, V., Pattwell, S.S., Ruberry, E.J., et al., 2010. The storm and stress of adolescence: insights from human imaging and mouse genetics. Dev. Psychobiol. 52, 225-235. https://doi.org/10.1002/dev.20447.

Conboy, L., Bisaz, R., Markram, K., Sandi, C., 2010. Role of NCAM in emotion and learning. Adv. Exp. Med. Biol. 663, 271-296. https://doi.org/10.1007/978-1-44191170-4_18.

Cordero, M.I., Just, N., Poirier, G.L., Sandi, C., 2016. Effects of paternal and peripubertal stress on aggression, anxiety, and metabolic alterations in the lateral septum. Eur. Neuropsychopharmacol 26, 357-367. https://doi.org/10.1016/j. euroneuro.2015.11.017.

Cordero, M.I., Poirier, G.L., Márquez, C., Veenit, V., Fontana, X., Salehi, B., et al., 2012. Evidence for biological roots in the transgenerational transmission of intimate partner violence. Transl. Psychiatry 2, e106. https://doi.org/10.1038/tp.2012.32.

De Kloet, E.R., 1991. Brain corticosteroid receptor balance and homeostatic control. Front. Neuroendocrinol. 12 (2), 95-164.

de Kloet, E.R., Karst, H., Joëls, M., 2008. Corticosteroid hormones in the central stress response: quick-and-slow. Front. Neuroendocrinol. 29, 268-272. https://doi.org/ 10.1016/j.yfrne.2007.10.002.

de Kloet, E.R., Meijer, O.C., de Nicola, A.F., de Rijk, R.H., Joëls, M., 2018. Importance of the brain corticosteroid receptor balance in metaplasticity, cognitive performance and neuro-inflammation. Front. Neuroendocrinol. 49, 124-145. https://doi.org/ 10.1016/j.yfrne.2018.02.003.

de Quervain, D., Schwabe, L., Roozendaal, B., 2017. Stress, glucocorticoids and memory: implications for treating fear-related disorders. Nat. Rev. Neurosci. 18, 7-19. https://doi.org/10.1038/nrn.2016.155.

Doyle, E., Nolan, P.M., Bell, R., Regan, C.M., 1992. Hippocampal NCAM180 transiently increases sialylation during the acquisition and consolidation of a passive avoidance response in the adult rat. J. Neurosci. Res. 31, 513-523. https://doi.org/10.1002/ jnr.490310315.

Gee, D.G., Casey, B.J., 2015. The impact of developmental timing for stress and recovery. Neurobiol. Stress 1, 184-194. https://doi.org/10.1016/j.ynstr.2015.02.001.

Gjerstad, J.K., Lightman, S.L., Spiga, F., 2018. Role of glucocorticoid negative feedback in the regulation of HPA axis pulsatility. Stress 21, 403-416. https://doi.org/ 10.1080/10253890.2018.1470238.

Gunnar, M.R., DePasquale, C.E., Reid, B.M., Donzella, B., Miller, B.S., 2019. Pubertal stress recalibration reverses the effects of early life stress in postinstitutionalized children. Proc. Natl. Acad. Sci. U.S.A. 116, 23984-23988. https://doi.org/10.1073/ pnas.1909699116.

Heim, C., Nemeroff, C.B., 2001. The role of childhood trauma in the neurobiology of mood and anxiety disorders: preclinical and clinical studies. Biol. Psychiatr. 49, 1023-1039. https://doi.org/10.1016/s0006-3223(01)01157-x.

Herman, J.P., Mueller, N.K., 2006. Role of the ventral subiculum in stress integration. Behav. Brain Res. 174, 215-224. https://doi.org/10.1016/j.bbr.2006.05.035.

Huzard, D., Vouros, A., Monari, S., Astori, S., Vasilaki, E., Sandi, C., 2020. Constitutive differences in glucocorticoid responsiveness are related to divergent spatial information processing abilities. Stress 23, 37-49. https://doi.org/10.1080, 10253890.2019.1625885.

Isgor, C., Kabbaj, M., Akil, H., Watson, S.J., 2004. Delayed effects of chronic variable stress during peripubertal-juvenile period on hippocampal morphology and on cognitive and stress axis functions in rats. Hippocampus 14, 636-648. https://doi org /10.1002/hipo.10207.

Jacobson, L., Sapolsky, R., 1991. The role of the hippocampus in feedback regulation of the hypothalamic-pituitary-adrenocortical axis. Endocr. Rev. 12, 118-134. https:// doi.org/10.1210/edrv-12-2-118.

Karatsoreos, I.N., McEwen, B.S., 2011. Psychobiological allostasis: resistance, resilience and vulnerability. Trends Cognit. Sci. 15, 576-584. https://doi.org/10.1016/j. tics.2011.10.005.

Kiss, J.Z., Muller, D., 2001. Contribution of the neural cell adhesion molecule to neuronal and synaptic plasticity. Rev. Neurosci. 12, 297-310.

Kovács, K.J., Makara, G.B., 1988. Corticosterone and dexamethasone act at different brain sites to inhibit adrenalectomy-induced adrenocorticotropin hypersecretion. Brain Res. 474, 205-210. https://doi.org/10.1016/0006-8993(88)90435-0.

Kumsta, R., Schlotz, W., Golm, D., Moser, D., Kennedy, M., Knights, N., et al., 2017. HPA axis dysregulation in adult adoptees twenty years after severe institutional deprivation in childhood. Psychoneuroendocrinology 86, 196-202. https://doi.org/ 10.1016/j.psyneuen.2017.09.021.

Latsko, M.S., Farnbauch, L.A., Gilman, T.L., Lynch, J.F., Jasnow, A.M., 2016. Corticosterone may interact with peripubertal development to shape adult resistance to social defeat. Horm. Behav. 82, 38-45. https://doi.org/10.1016/j. yhbeh.2016.04.009.

López-Fernández, M.A., Montaron, M.-F., Varea, E., Rougon, G., Venero, C., Abrous, D. N., Sandi, C., 2007. Upregulation of polysialylated neural cell adhesion molecule in the dorsal hippocampus after contextual fear conditioning is involved in long-term memory formation. J. Neurosci. 27, 4552-4561. https://doi.org/10.1523/ JNEUROSCI.0396-07.2007.

Lupien, S.J., McEwen, B.S., Gunnar, M.R., Heim, C., 2009. Effects of stress throughout the lifespan on the brain, behaviour and cognition. Nat. Rev. Neurosci. 10, 434-445. https://doi.org/10.1038/nrn2639.

Márquez, C., Nadal, R., Armario, A., 2004. The hypothalamic-pituitary-adrenal and glucose responses to daily repeated immobilisation stress in rats: individual differences. Neuroscience 123, 601-612. https://doi.org/10.1016/j. neuroscience.2003.10.016.

Márquez, C., Poirier, G.L., Cordero, M.I., Larsen, M.H., Groner, A., Marquis, J., Magistretti, P.J., Trono, D., Sandi, C., 2013. Peripuberty stress leads to abnormal aggression, altered amygdala and orbitofrontal reactivity and increased prefrontal 
MAOA gene expression. Transl. Psychiatry 3, e216. https://doi.org/10.1038/ tp.2012.144.

McCormick, C.M., Green, M.R., Simone, J.J., 2017. Translational relevance of rodent models of hypothalamic-pituitary-adrenal function and stressors in adolescence. Neurobiol. Stress 6, 31-43. https://doi.org/10.1016/j.ynstr.2016.08.003.

McCormick, C.M., Mathews, I.Z., 2010. Adolescent development, hypothalamicpituitary-adrenal function, and programming of adult learning and memory. Prog. Neuro-Psychopharmacol. Biol. Psychiatry 34, 756-765. https://doi.org/10.1016/j. pnpbp.2009.09.019.

McEwen, B.S., Nasca, C., Gray, J.D., 2016. Stress effects on neuronal structure: Hippocampus, amygdala, and prefrontal cortex. Neuropsychopharmacology 41, 3-23. https://doi.org/10.1038/npp.2015.171.

Molet, J., Maras, P.M., Avishai-Eliner, S., Baram, T.Z., 2014. Naturalistic rodent models of chronic early-life stress. Dev. Psychobiol. 56, 1675-1688. https://doi.org/ 10.1002/dev.21230.

Montaron, M.F., Piazza, P.V., Aurousseau, C., Urani, A., Le Moal, M., Abrous, D.N., 2003. Implication of corticosteroid receptors in the regulation of hippocampal structural plasticity. Eur. J. Neurosci. 18, 3105-3111. https://doi.org/10.1111/j.14609568.2003.03048.x

Moser, M.B., Moser, E.I., Forrest, E., Andersen, P., Morris, R.G., 1995. Spatial learning with a minislab in the dorsal hippocampus. Proc. Natl. Acad. Sci. U.S.A. 92, 9697-9701. https://doi.org/10.1073/pnas.92.21.9697.

Murphy, K.J., O'Connell, A.W., Regan, C.M., 1996. Repetitive and transient increases in hippocampal neural cell adhesion molecule polysialylation state following multitrial spatial training. J. Neurochem. 67, 1268-1274. https://doi.org/10.1046/j.1471 4159.1996.67031268.x.

Myers, B., McKlveen, J.M., Herman, J.P., 2014. Glucocorticoid actions on synapses, circuits, and behavior: implications for the energetics of stress. Front. Neuroendocrinol. 35, 180-196. https://doi.org/10.1016/j.yfrne.2013.12.003.

Nacher, J., Gómez-Climent, M.Á., McEwen, B., 2004. Chronic non-invasive glucocorticoid administration decreases polysialylated neural cell adhesion molecule expression in the adult rat dentate gyrus. Neurosci. Lett. 370, 40-44. https://doi. org/10.1016/j.neulet.2004.07.062.

Oomen, C.A., Soeters, H., Audureau, N., Vermunt, L., van Hasselt, F.N., Manders, E.M.M., et al., 2010. Severe early life stress hampers spatial learning and neurogenesis, but improves hippocampal synaptic plasticity and emotional learning under high-stress conditions in adulthood. J. Neurosci. https://doi.org/10.1523/JNEUROSCI.0247. 10.2010 .

Papilloud, A., Guillot de Suduiraut, I., Zanoletti, O., Grosse, J., Sandi, C., 2018. Peripubertal stress increases play fighting at adolescence and modulates nucleus accumbens CB1 receptor expression and mitochondrial function in the amygdala. Transl. Psychiatry 8. https://doi.org/10.1038/s41398-018-0215-6, 156-12.

Papilloud, A., Veenit, V., Tzanoulinou, S., Riccio, O., Zanoletti, O., Guillot de Suduiraut, I., et al., 2019. Peripubertal stress-induced heightened aggression: modulation of the glucocorticoid receptor in the central amygdala and normalization by mifepristone treatment. Neuropsychopharmacology 44, 674-682. https://doi. org/10.1038/s41386-018-0110-0.

Paus, T., Keshavan, M., Giedd, J.N., 2008. Why do many psychiatric disorders emerge during adolescence? Nat. Rev. Neurosci. 9, 947-957. https://doi.org/10.1038/ nrn2513.

Pham, K., Nacher, J., Hof, P.R., McEwen, B.S., 2003. Repeated restraint stress suppresse neurogenesis and induces biphasic PSA-NCAM expression in the adult rat dentate gyrus. Eur. J. Neurosci. 17, 879-886. https://doi.org/10.1046/j.1460 9568.2003.02513.x.

Poirier, G.L., Imamura, N., Zanoletti, O., Sandi, C., 2014. Social deficits induced by peripubertal stress in rats are reversed by resveratrol. J. Psychiatr. Res. 57, 157-164. https://doi.org/10.1016/j.jpsychires.2014.05.017.

Quirarte, G.L., Roozendaal, B., McGaugh, J.L., 1997. Glucocorticoid enhancement of memory storage involves noradrenergic activation in the basolateral amygdala. Proc. Natl. Acad. Sci. U.S.A. 94, 14048-14053. https://doi.org/10.1073/ pnas.94.25.14048.

Reul, J.M., Sutanto, W., van Eekelen, J.A., Rothuizen, J., de Kloet, E.R., 1990. Central action of adrenal steroids during stress and adaptation. Adv. Exp. Med. Biol. 274, 243-256. https://doi.org/10.1007/978-1-4684-5799-5_15.

Rodríguez, J.J., Montaron, M.F., Petry, K.G., Aurousseau, C., Marinelli, M., Premier, S. Rougon, G., Le Moal, M., Abrous, D.N., 1998. Complex regulation of the expression of the polysialylated form of the neuronal cell adhesion molecule by glucocorticoids in the rat hippocampus. Eur. J. Neurosci. 10, 2994-3006. https://doi.org/10.1046/ j.1460-9568.1998.00316.x.

Romeo, R.D., Patel, R., Pham, L., So, V.M., 2016. Adolescence and the ontogeny of the hormonal stress response in male and female rats and mice. Neurosci. Biobehav. Rev. 70, 206-216. https://doi.org/10.1016/j.neubiorev.2016.05.020.

Rutishauser, U., 2008. Polysialic acid in the plasticity of the developing and adult vertebrate nervous system. Nat. Rev. Neurosci. 9, 26-35. https://doi.org/10.1038/ nrn2285.

Salehi, B., Cordero, M.I., Sandi, C., 2010. Learning under stress: the inverted-U-shape function revisited. Learn. Mem. 17, 522-530. https://doi.org/10.1101/lm.1914110.

Sandi, C., 2013. Stress and Cognition, vol. 4. Wiley Interdiscip Rev Cogn Sci, pp. 245-261. https://doi.org/10.1002/wcs.1222.

Sandi, C., 2011. Glucocorticoids act on glutamatergic pathways to affect memory processes. Trends Neurosci. 34, 165-176. https://doi.org/10.1016/j. tins.2011.01.006.

Sandi, C., 2004. Stress, cognitive impairment and cell adhesion molecules. Nat. Rev. Neurosci. 5, 917-930. https://doi.org/10.1038/nrn1555.
Sandi, C., Cordero, M.I., Merino, J.J., Kruyt, N.D., Regan, C.M., Murphy, K.J., 2004. Neurobiological and endocrine correlates of individual differences in spatial learning ability. Learn. Mem. 11, 244-252. https://doi.org/10.1101/lm.73904.

Sandi, C., Loscertales, M., Guaza, C., 1997. Experience-dependent facilitating effect of corticosterone on spatial memory formation in the water maze. Eur. J. Neurosci. 9, 637-642. https://doi.org/10.1111/j.1460-9568.1997.tb01412.x.

Sandi, C., Merino, J.J., Cordero, M.I., Kruyt, N.D., Murphy, K.J., Regan, C.M., 2003. Modulation of hippocampal NCAM polysialylation and spatial memory consolidation by fear conditioning. Biol. Psychiatr. 54, 599-607. https://doi.org/10.1016/s00063223(03)00182-3.

Sandi, C., Merino, J.J., Cordero, M.I., Touyarot, K., Venero, C., 2001. Effects of chronic stress on contextual fear conditioning and the hippocampal expression of the neural cell adhesion molecule, its polysialylation, and L1. Neuroscience 102, 329-339. https://doi.org/10.1016/s0306-4522(00)00484-x.

Schenk, F., 1985. Development of place navigation in rats from weaning to puberty. Behav. Neural. Biol. 43, 69-85. https://doi.org/10.1016/s0163-1047(85)91510-9.

Sheth, C., McGlade, E., Yurgelun-Todd, D., 2017. Chronic stress in adolescents and its neurobiological and psychopathological consequences: an RDoC perspective (Thousand Oaks). Chronic Stress 1. https://doi.org/10.1177/2470547017715645, 247054701771564.

Spear, L.P., 2000. The adolescent brain and age-related behavioral manifestations Neurosci. Biobehav. Rev. 24, 417-463.

Sterlemann, V., Rammes, G., Wolf, M., Liebl, C., Ganea, K., Müller, M.B., et al., 2010. Chronic social stress during adolescence induces cognitive impairment in aged mice. Hippocampus 20, 540-549. https://doi.org/10.1002/hipo.20655.

Suri, D., Veenit, V., Sarkar, A., Thiagarajan, D., Kumar, A., Nestler, E.J., 2013. Early stress evokes age-dependent biphasic changes in hippocampal neurogenesis, BDNF expression, and cognition. Biol. Psychiatr. 73, 658-666. https://doi.org/10.1016/j. biopsych.2012.10.023.

Toledo-Rodriguez, M., Sandi, C., 2011. Stress during adolescence increases novelty seeking and risk-taking behavior in male and female rats. Front. Behav. Neurosci. 5, 17. https://doi.org/10.3389/fnbeh.2011.00017.

Tsoory, M., Guterman, A., Richter-Levin, G., 2008. Exposure to stressors during juvenility disrupts development-related alterations in the PSA-NCAM to NCAM expression ratio: potential relevance for mood and anxiety disorders. Neuropsychopharmacology 33, 378-393. https://doi.org/10.1038/sj.npp.1301397.

Tzanoulinou, S., García-Mompó, C., Castillo-Gómez, E., Veenit, V., Nacher, J., Sandi, C. 2014a. Long-term behavioral programming induced by peripuberty stress in rats is accompanied by GABAergic-related alterations in the Amygdala. PloS One 9, e94666. https://doi.org/10.1371/journal.pone.0094666.

Tzanoulinou, S., García-Mompó, C., Riccio, O., Grosse, J., Zanoletti, O., Dedousis, P., et al., 2016. Neuroligin-2 expression in the prefrontal cortex is involved in attention deficits induced by peripubertal stress. Neuropsychopharmacology 41, 751-761. https://doi.org/10.1038/npp.2015.200.

Tzanoulinou, S., Riccio, O., de Boer, M.W., Sandi, C., 2014b. Peripubertal stress-induced behavioral changes are associated with altered expression of genes involved in excitation and inhibition in the amygdala. Transl. Psychiatry 4, e410. https://doi. org/10.1038/tp.2014.54.

Tzanoulinou, S., Sandi, C., 2017. The programming of the social brain by stress during childhood and adolescence: from rodents to humans. Curr. Top. Behav. Neurosci. 30, 411-429. https://doi.org/10.1007/7854 2015430.

Tzanoulinou, S., Sandi, C., 2016. The programming of the social brain by stress during childhood and adolescence: from rodents to humans. Curr. Top. Behav. Neurosci. 1-19. https://doi.org/10.1007/7854 2015430.

Varbanov, H., Dityatev, A., 2017. Regulation of extrasynaptic signaling by polysialylated NCAM: impact for synaptic plasticity and cognitive functions. Mol. Cell. Neurosci. 81, 12-21. https://doi.org/10.1016/j.mcn.2016.11.005.

Veenema, A.H., 2009. Early life stress, the development of aggression and neuroendocrine and neurobiological correlates: what can we learn from animal models? Front. Neuroendocrinol. 30, 497-518. https://doi.org/10.1016/j. yfrne.2009.03.003.

Veenit, V., Cordero, M.I., Tzanoulinou, S., Sandi, C., 2013. Increased corticosterone in peripubertal rats leads to long-lasting alterations in social exploration and aggression. Front. Behav. Neurosci. 7, 26. https://doi.org/10.3389/ fnbeh.2013.00026.

Veenit, V., Riccio, O., Sandi, C., 2014. CRHR1 links peripuberty stress with deficits in social and stress-coping behaviors. J. Psychiatr. Res. 53, 1-7. https://doi.org/ 10.1016/j.jpsychires.2014.02.015.

Venero, C., Herrero, A.I., Touyarot, K., Cambon, K., López-Fernández, M.A., Berezin, V., et al., 2006. Hippocampal up-regulation of NCAM expression and polysialylation plays a key role on spatial memory. Eur. J. Neurosci. 23, 1585-1595. https://doi. org/10.1111/j.1460-9568.2006.04663.x.

Venero, C., Tilling, T., Hermans-Borgmeyer, I., Schmidt, R., Schachner, M., Sandi, C., 2002. Chronic stress induces opposite changes in the mRNA expression of the cell adhesion molecules NCAM and L1. Neuroscience 115, 1211-1219. https://doi.org/ 10.1016/s0306-4522(02)00543-2.

Walker, S.E., Sandi, C., 2018. Long-term programing of psychopathology-like behaviors in male rats by peripubertal stress depends on individual's glucocorticoid responsiveness to stress. Stress 21, 433-442. https://doi.org/10.1080/ 10253890.2018.1435639.

Walker, S.E., Wood, T.C., Cash, D., Mesquita, M., Williams, S.C.R., Sandi, C., 2018. Alterations in brain microstructure in rats that develop abnormal aggression following peripubertal stress. Eur. J. Neurosci. 48, 1818-1832. https://doi.org/ 10.1111/ejn.14061.

Walker, S.E., Zanoletti, O., Guillot de Suduiraut, I., Sandi, C., 2017. Constitutive differences in glucocorticoid responsiveness to stress are related to variation in 
aggression and anxiety-related behaviors. Psychoneuroendocrinology 84, 1-10. https://doi.org/10.1016/j.psyneuen.2017.06.011. 